\title{
The Origin of Corticospinal Projections from the Premotor Areas in the Frontal Lobe
}

\author{
Richard P. Dum and Peter L. Strick \\ Research Service, Veterans Administration Medical Center and Departments of Neurosurgery and Physiology, SUNY \\ Health Science Center at Syracuse, Syracuse, New York 13210
}

We determined the origin of corticospinal neurons in the frontal lobe. These neurons were labeled by retrograde transport of tracers after injections into either the dorsolateral funiculus at the second cervical segment or the gray matter of the spinal cord throughout the cervical enlargement. Using retrograde transport of tracer from the arm area of the primary motor cortex, we defined the arm representation in each premotor area in another set of animals.

We found that corticospinal projections to cervical segments of the spinal cord originate from the primary motor cortex and from the 6 premotor areas in the frontal lobe. These are the same premotor areas that project directly to the arm area of the primary motor cortex. The premotor areas are located in parts of cytoarchitectonic area 6 on the lateral surface and medial wall of the hemisphere, as well as in subfields of areas 23 and 24 In the cingulate sulcus. The total number of corticospinal neurons in the arm representations of the premotor areas equals or exceeds the total number in the arm representation of the primary motor cortex. The premotor areas collectively comprise more than $60 \%$ of the cortical area in the frontal lobe that projects to the spinal cord. Like the primary motor cortex, each of the premotor areas contains local regions that have a high density of corticospinal neurons.

These observations indicate that a substantial component of the corticospinal system originates from the premotor areas in the frontal lobe. Each of the premotor areas has direct access to the spinal cord, and as a consequence, each has the potential to influence the generation and control of movement independently of the primary motor cortex. These findings raise serious questions about the utility of viewing the primary motor cortex as the "upper motoneuron" or "final common pathway" for the central control of movement.

The term "premotor cortex" was originally applied to that portion of the frontal agranular cortex located rostral to the primary motor cortex (Fulton, 1935). Fulton stated that "strictly speaking, the premotor area is restricted to the superior part of area

\footnotetext{
Received July 2, 1990; revised Oct. 4, 1990; accepted Oct. 9, 1990.

This work was initiated by support from the Eastern Paralyzed Veterans Association and sustained by funds from the Veterans Administration Medical Research Service and Rehabilitation Research and Development Service and by U.S. Public Health Service Grants 02957 and 24328 . We wish to thank Mr. Mike Page for development of computer programs, Ms. Mary Lou Cherney and Sarah Fitzpatrick for their expert technical assistance, and Drs. Kenneth D. Hutchins and Anthony M. Martino for their assistance in some of the initial experiments.

Correspondence should be addressed to Dr. Peter L. Strick, Research Service (151), Veterans Administration Medical Center, Syracuse, NY 13210.

Copyright (C) 1991 Society for Neuroscience 0270-6474/91/110667-23\$03.00/0
}

6a" (Fulton, 1949, p 318) and indicated that it was related to the control of fore- and hindlimb movements. His review of this subject suggests that he considered the portion of area $6 \mathrm{a}$ inferior to the arcuate sulcus as the premotor area for oral-facial movements (Fulton, 1949). It is clear that Fulton's premotor cortex included that part of area 6 that lies on the medial wall of the hemisphere. Subsequently, this region was defined as the supplementary motor area (SMA; Penfield and Welch, 1951; Woolscy ct al., 1952).

Classically, the premotor cortex has been viewed as functionally distinct from the primary motor cortex and as a center for the integration of complex skilled movements (Campbell, 1905; Fulton and Kennard, 1934; Jacobsen, 1934, 1935). Lesions of the premotor cortex in subhuman primates caused "a disorganization of more highly integrated voluntary movements producing a state akin to apraxia in man" (Fulton, 1935, p 313; see also Jacobsen, 1934, 1935). Electrical stimulation at different sites in the premotor cortex of humans and monkeys evoked movements of proximal and distal body parts (for historical review, see Fulton and Kennard, 1934; Fulton, 1949; Penfield and Welch, 1951; Woolsey et al., 1952; Wiesendanger, 1981). However, when the primary motor cortex was removed, stimulation of the premotor cortex could evoke movements of only proximal body parts (e.g., Vogt and Vogt, 1919; Foerster, 1936; Wiesendanger et al., 1973). Based on early anatomical studies, the premotor area was thought to lack substantial projections to the spinal cord (Fulton, 1949). These and other observations were consistent with a hierarchical concept of the cortical motor areas in which the premotor cortex was at the highest level (Campbell, 1905) and participated in the generation and control of distal movement through its projections to the primary motor cortex (area 4).

Since these early studies, there has been considerable progress in defining the anatomical location of the premotor cortex (Matsumura and Kubota, 1979; Muakkassa and Strick, 1979; Godschalk et al., 1984; Strick, 1985; Leichnetz, 1986; Ghosh et al., 1987; Dum and Strick, 1990). Based on the hierarchical concept, we have operationally defined the premotor cortex as that region in the frontal lobe that has direct projections to the primary motor cortex (area 4). According to this definition, the premotor cortex is heterogeneous and is comprised of multiple, spatially separate "premotor areas" (Pandya and Kuypers, 1969; Pandya and Vignolo, 1971; Künzle, 1978; Matsumura and Kubota, 1979; Muakkassa and Strick, 1979; Godschalk et al., 1984; Strick, 1985; Leichnetz, 1986; Ghosh et al., 1987). For example, the arm area of the primary motor cortex receives inputs from 3 regions within area 6: (1) the SMA on the medial wall of the hemisphere, (2) the arcuate premotor area (APA) in and around the caudal bank of the arcuate sulcus, and (3) the region in and 
adjacent to the superior precentral sulcus (SPCS). The primary motor cortex also receives inputs from 2 regions of the frontal lobe that lie within subfields of areas 23 and 24 in the banks of the cingulate sulcus (Künzle, 1978; Muakkassa and Strick, 1979; Godschalk et al., 1984; Strick, 1985; Leichnetz, 1986; Ghosh et al., 1987; Dum and Strick, 1990) and have been termed the rostral and caudal cingulate motor areas (CMAr and CMAc; Hutchins et al., 1988; Dum and Strick, 1989). Thus, the anatomical organization of the premotor cortex is more complicated than previously recognized (for reviews, see Fulton, 1949; Woolsey et al., 1952; Humphrey, 1979; Wiesendanger, 1981; Wise, 1985).

In recent studies, we and others have begun to examine some of the afferent inputs to each of the premotor areas. For example, these studies have shown that the arm representation in the SMA receives projections from regions of the ventrolateral thalamus that are the site of termination of efferents from the basal ganglia (e.g., Schell and Strick, 1984; Wiesendanger and Wiesendanger, 1985a). In contrast, the arm representation in the APA receives projections from regions of the ventrolateral thalamus that are the site of termination for a set of cerebellar efferents (e.g., Schell and Strick, 1984; Matelli et al., 1989; Orioli and Strick, 1989). Another set of cerebellar efferents projects to regions of the ventrolateral thalamus that innervate the portion of the arm area of the primary motor cortex located on the crest of the precentral gyrus (Schell and Strick, 1984; Wiesendanger and Wiesendanger, 1985a,b; Matelli et al., 1989; Orioli and Strick, 1989). Based on these and other observations, we have proposed that each premotor area, along with the primary motor cortex, is a nodal point for a distinct set of afferent inputs from subcortical motor nuclei, such as the ccrebellum and basal ganglia, and from cortical areas in the parietal lobe (Schell and Strick, 1984; Galyon and Strick, 1985; Strick, 1988; Dum and Strick, 1990). Thus, we view these cortical motor areas as components of anatomically distinct systems for the central control of movement (Schell and Strick, 1984; Strick, 1988; Dum and Strick, 1990). According to this view, these systems operate largely in parallel, but interact at the cortical level where the motor areas are densely interconnected.

An important consequence of our proposal is that it places the premotor areas at the same level of hierarchical organization as the primary motor cortex. This suggestion raises an important issue: To what extent do the premotor areas have outputs that are independent of the primary motor cortex? In fact, studies using retrograde transport techniques have provided evidence that some of the premotor areas project directly to the spinal cord (Catsman-Berrevoets and Kuypers, 1976; Biber et al., 1978; Murray and Coulter, 1981; Macpherson et al., 1982; Toyoshima and Sakai, 1982; Martino and Strick, 1987; Hutchins et al., 1988; Dum and Strick, 1989, 1990; Keizer and Kuypers, 1989). However, these studies did not specifically examine the areal extent and density of corticospinal projections from each of the premotor areas. Furthermore, the relationship between the premotor areas in the cingulate sulcus and the origin of corticospinal projections from this region has not been examined in detail.

To investigate these issues, we labeled cortical neurons that project to the spinal cord using retrograde transport techniques. We focused specifically on the origin of corticospinal projections from the premotor areas to cervical segments of the spinal cord. The size, number, density, and areal extent of corticospinal neurons in the premotor areas were compared with those found in the primary motor cortex. Finally, in another set of animals, we defined the arm representation in each premotor area using the retrograde transport of tracer from the arm area of the primary motor cortex.

Our observations indicate that corticospinal projections to cervical segments of the spinal cord originate from the same areas of the premotor cortex that project to the arm area of the primary motor cortex. In fact, several quantitative measures indicate that the size of the corticospinal system from the premotor areas equals or exceeds that from the primary motor cortex. These findings raise serious questions about the utility of viewing the primary motor cortex as the "upper motoneuron" or "final common pathway" for the central control of movement.

Short reports of some of the data presented in this article have appcarcd previously (Dum and Strick, 1988).

\section{Materials and Methods}

\section{Surgical procedures}

We used retrograde transport of horseradish peroxidase (HRP) or wheat germ agglutinin conjugated to HRP (WGA-HRP) to determine the origin of projections from the frontal lobe to the spinal cord. These studies were performed on 6 pig-tailed macaques (Macaca nemestrina; 4.0$5.75 \mathrm{~kg}$ ). An animal was prepared for surgery by restricting food and water for $6-12 \mathrm{hr}$ and by pretreament with dexamethasone $(0.5 \mathrm{mg} / \mathrm{kg}$, i.m., given 6 and $12 \mathrm{hr}$ prior to surgery). The animal was initially anesthetized with ketamine $(10 \mathrm{mg} / \mathrm{kg}, \mathrm{i} . \mathrm{m}$.) and Nembutal $(20 \mathrm{mg} / \mathrm{kg}$ i.p.). Additional doses of ketamine (5-10 mg/kg, i.m.) and/or Nembutal $(5 \mathrm{mg} / \mathrm{kg}, \mathrm{i} . \mathrm{v}$.) were given as needed to maintain anesthesia. During surgery, each animal was given atropine $(0.1 \mathrm{mg} / \mathrm{kg})$ and antibiotics (Kefzol, $25 \mathrm{mg} / \mathrm{kg}$, every $6 \mathrm{hr}$; Oxycillin, $25 \mathrm{mg} / \mathrm{kg}$, every $4 \mathrm{hr}$; gentamicin, $1 \mathrm{mg} / \mathrm{kg}$, every $8 \mathrm{hr}$ ) and was hydrated with intravenous fluids (lactated Ringer's solution, approximately $10-20 \mathrm{cc} / \mathrm{hr}$ ). Respiration, heart rate, body temperature, and urinary output were monitored. Body temperature was maintained with a heating pad.

All surgical procedures were performed using sterile techniques. A laminectomy was performed over the appropriate segmental levels of the cervical spinal cord. The dura was opened, and the surface of the spinal cord was kept moist using warmed surgical-grade silicone. Thirty to $60 \mathrm{~min}$ prior to opening the dura, we gave a bolus of mannitol (1.5$2 \mathrm{gm} / \mathrm{kg}$ of a $25 \%$ solution, i.v.). The appropriate segmental level for injections was determined by identifying vertebrae and dorsal root entry zones (Hartman and Straus, 1933; Hill, 1974). We used 2 protocols to inject the spinal cord. In the first protocol $(n=2)$, we transected the dorsolateral funiculus at the second cervical segment (C2) using fine scissors. Then, crystalline HRP ( $34 \mathrm{mg}$; Sigma, Type VI) was packed into the lesion, and the area was covered with Gelfoam.

In the second protocol $(n=2)$, we injected WGA-HRP (Sigma; $2 \%$ in $0.5 \mathrm{M} \mathrm{NaCl}$ with $0.1 \mathrm{M}$ mannose added) into the gray matter of specific cervical segments using a $1-\mu \mathrm{l}$ Hamilton syringe with a 32-gauge needle. A $2 \%$ solution of WGA-HRP was used because prior studies have shown that this concentration of tracer is not incorporated and transported by fibers of passage (for complete discussion, see Mesulam, 1982; Gibson et al., 1984). Injection sites were spaced $1-1.5 \mathrm{~mm}$ apart, except to avoid blood vessels. We angled the injection needle to enter the spinal cord by passing through the dorsal column. This was done to avoid damage to the dorsolateral funiculus, where most of the corticospinal tract descends. We made injections $(0.05 \mu \mathrm{l})$ at 2 depths along each track in order to completely fill the gray matter of the spinal cord with tracer. Following each injection, the needle was left in place at least 3 min and then withdrawn.

Upon the completion of either injection protocol, the spinal cord was covered with a thin sheet of surgical-grade Silastic and Gelfoam. The wound was closed in layers. The animal was returned to its home cage, and its recovery was carefully monitored throughout the survival period.

In 2 animals, multiple injections ( 8 tracks, $0.05 \mu \mathrm{l} / \mathrm{site}$ ) of WGA-HRP were made into the arm area of the primary motor cortex. The surgical procedures we employed were identical to those detailed in previous publications from this laboratory (Schell and Strick, 1984; Orioli and Strick, 1989). Tracer injections were made throughout the arm area of the primary motor cortex and included both the precentral gyrus and the anterior bank of the central sulcus (Woolsey et al., 1952). Injections 
into the gyrus wcre placed at a depth of $1.5 \mathrm{~mm}$, and those into the sulcus were placed at $1-\mathrm{mm}$ intervals at depths from 1.5 to $4.5 \mathrm{~mm}$.

After a 3-5-d survival period, all animals were reanesthetized with ketamine $(20 \mathrm{mg} / \mathrm{kg}$, i.m.) and Nembutal $(36 \mathrm{mg} / \mathrm{kg}$, i.p.). They were perfused transcardially using a 4-stage procedure (see also Rosene and Mesulam, 1978; Mesulam, 1982). The perfusates for animals with injections into the spinal cord included (1) $0.1 \mathrm{~m}$ phosphate buffer $(\mathrm{pH}$, 7.4), (2) $2.5 \%$ glutaraldehyde in $0.1 \mathrm{~m}$ phosphate buffer, (3) $2.5 \%$ glutaraldehyde in $0.1 \mathrm{~m}$ phosphate buffer with $10 \%$ glycerin, and (4) $0.1 \mathrm{M}$ phosphate buffer with $10 \%$ glycerin. Paraformaldehyde (2.5\%) was substituted for the glutaraldehyde in the perfusates used in the animal with injections into the primary motor cortex. Following the perfusion, the brain was blocked in situ while the skull was held in a stereotaxic frame. The brain block included the arcuate sulcus rostrally and the intraparietal sulcus caudally. Then, both the brain and the spinal cord were stored in $0.1 \mathrm{M}$ phosphate buffer with $10-20 \%$ glycerin at $4^{\circ} \mathrm{C}$.

\section{Histological Procedures}

Four to $7 \mathrm{~d}$ later, the brain block was quick frozen in cold isopentane (Rosene et al., 1986). Serial coronal sections $(50 \mu \mathrm{m})$ of this block were cut on a microtome using a controlled freezing stage. Every tenth section was postfixed and processed for cytoarchitecture according to the technique outlined by Gower (in Mesulam, 1982). The remaining sections were processed for HRP using tetramethylbenzidine (TMB) as the substrate (Mesulam, 1982; Gibson et al., 1984). Spinal cord regions containing the injection sites also were sectioned at $50 \mu \mathrm{m}$. Every tenth section of spinal cord was postfixed and processed for cytoarchitecture, and the remaining sections were processed for HRP using the TMB procedure

\section{Analytical procedures}

At least every fourth section through the frontal cortex was examined for labeled neurons under bright-field and/or dark-field/polarized illumination. The outline of the section and the location of each labeled neuron was charted on an $x-y$ plotter driven by linear potentiometers coupled to the microscope stage. At least every tenth spinal cord section through the injection site was examined and charted in a similar manner to determine the spread of HRP or WGA-HRP. The charts of individual sections were then entered into an IBM AT computer using a highresolution digitizing tablet. These charts were used to reconstruct the distribution of labeled neurons in the frontal lobe. Two different types of reconstructions were generated: a flattened view of the lateral hemisphere along with the medial surface and a 3-dimensional view of the caudal bank of the arcuate sulcus.

Flattened reconstruction. In confirmation of prior studies, we found that all corticospinal neurons were located in layer V (e.g., Biber et al., 1978: Murray and Coulter, 1981; Toyoshima and Sakai, 1982; Nudo and Masterton, 1990). Therefore, we produced flattened reconstructions (Fig. 1) of the frontal lobe by "straightening" this layer. To do this, the charts of coronal sections were aligned on the junction of the medial wall with the lateral surface of the hemisphere, and this point of alignment was kept fixed. Then, layer V on the medial wall of the hemisphere (i.e., the superior frontal gyrus, cingulate sulcus, and cingulate gyrus) was unfolded and reflected upward, and the lateral surface of the hemisphere was unfolded and reflected downward. The anterior bank of the central sulcus also was included in the flattened reconstructions. To display this portion of the primary motor cortex in an appropriate perspective, it was angled with respect to the lateral convexity. The angle chosen was perpendicular to the average angle between the central sulcus and the midline. To prevent distortion of surface features of the lateral convexity, we did not flatten 2 sulci: the superior precentral and the arcuate. Labeled neurons in these regions were plotted on the surface directly over their respective sulci on the flattened reconstructions.

$3-D$ reconstruction. The section outlines from the charts of coronal sections through the arcuate sulcus were carefully aligned. Then, the outline of the caudal bank of the arcuate sulcus and the position of labeled neurons in this region of the sulcus were digitized on an $x-y$ tablet. Computer programs allowed us to rotate the reconstructed sulcus in any of the 3 major planes and view the sulcus from an optimal angle (see Fig. 10).

Density analysis. The flattened layer $\mathrm{V}$ from each coronal section was divided into $200-\mu \mathrm{m}$ bins. The number of cells in each bin was counted and color coded. The lightest colors (white and yellow) were assigned to the bins with the most cells and represent approximately the upper
$10 \%$ of the bins containing labeled neurons. These were considered highdensity bins. The locations of the color-coded bins were then plotted on a flattened reconstruction of the frontal lobe (see above).

Determining cell density for bins located in the central sulcus was somewhat more complicated because it traverses the lateral hemisphere at an angle to the midline. Consequently, coronal sections through this sulcus cut layer $\mathrm{V}$ at an angle and effectively sampled a larger width of the layer. To correct for this sampling bias, the number of cells in each bin in the central sulcus was multiplied by the cosine of the angle formed by the sulcus and the midline of the hemisphere. Because the sulcus curves, the average angle formed by 3 sections spanning the one under analysis was used for this calculation. No other stereological corrections were applied to our data.

A third procedure was used to determine the density of labeled neurons in the caudal bank of the arcuate sulcus. We rotated the 3-dimensional reconstruction of the sulcus to view it normal to its caudal bank. This region was then overlaid with a $200-\mu \mathrm{m}$ square grid. The labeled neurons within each grid square were counted to determine the number of cells per bin.

Cell size analysis. Labeled cells were viewed at $500 \times$ and measured on a digitizing tablet using a camera lucida attachment. The size of pyramidal-shaped cells was represented by the average of their maximum and minimum diameters. Unless otherwise noted, only cells cut parallel to their long axes were measured. All measured cells contained a complete nucleus and/or had prominent apical and basal dendrites. No corrections were made for tissue shrinkage.

\section{Results}

\section{Cytoarchitecture of premotor areas on the medial wall}

Before presenting our observations on the topographic distribution of corticospinal neurons, we will describe some of the features that characterize the cytoarchitecture in the region of the premotor areas. There have been numerous descriptions of the architectonics in this region (e.g., Brodmann, 1905; Vogt and Vogt, 1919; von Bonin and Bailey, 1947; Matelli et al., 1985; Barbas and Pandya, 1987; Vogt et al., 1987). However, the cytoarchitecture of the cortex within the motor areas on the medial wall of the hemisphere has not been fully described in prior reports. We have focused our analysis on those regions on the medial wall that project to cervical segments of the spinal cord. These cortical areas are located largely caudal to the arcuate sulcus and rostral to the central sulcus. We will not describe the architectonics of the cortex on the lateral surface of the hemisphere because several recent studies have been published on this subject (e.g., Sessle and Wiesendanger, 1982; Weinrich and Wise, 1982; Matelli et al., 1985; Kurata and Tanji, 1986; Barbas and Pandya, 1987).

\section{Superior frontal gyrus}

Portions of areas 4 and 6 are found on the superior frontal gyrus on the medial wall of the hemisphere (Figs. $1 ; 2 A, D ; 3$ ). Four features characterize these cortical areas and allow them to be distinguished from others on the medial wall: (1) areas 4 and 6 have the thickest cortex on the medial wall, (2) the size of pyramidal-shaped cells increases with depth in layer III, (3) a granular layer IV is absent, and (4) layer VI is particularly thick in areas 4 and 6 . The border between areas 4 and 6 is not sharp. However, area 6 on the medial wall of the hemisphere, like on the lateral surface, is distinguished from area 4 by the greater number and size of giant pyramidal cells in layer $V$ of area 4 (Fig. 2A,D; see also Vogt and Vogt, 1919; Wise and Tanji, 1981; Macpherson et al., 1982; Sessle and Wiesendanger, 1982; Weinrich and Wise, 1982; Kurata and Tanji, 1986; Mitz and Wise, 1987). The border between these areas on the superior frontal gyrus lies approximately $7-8 \mathrm{~mm}$ caudal to the genu of the arcuate sulcus (Figs. 1,3). Ventrally, both areas 6 and 4 extend 


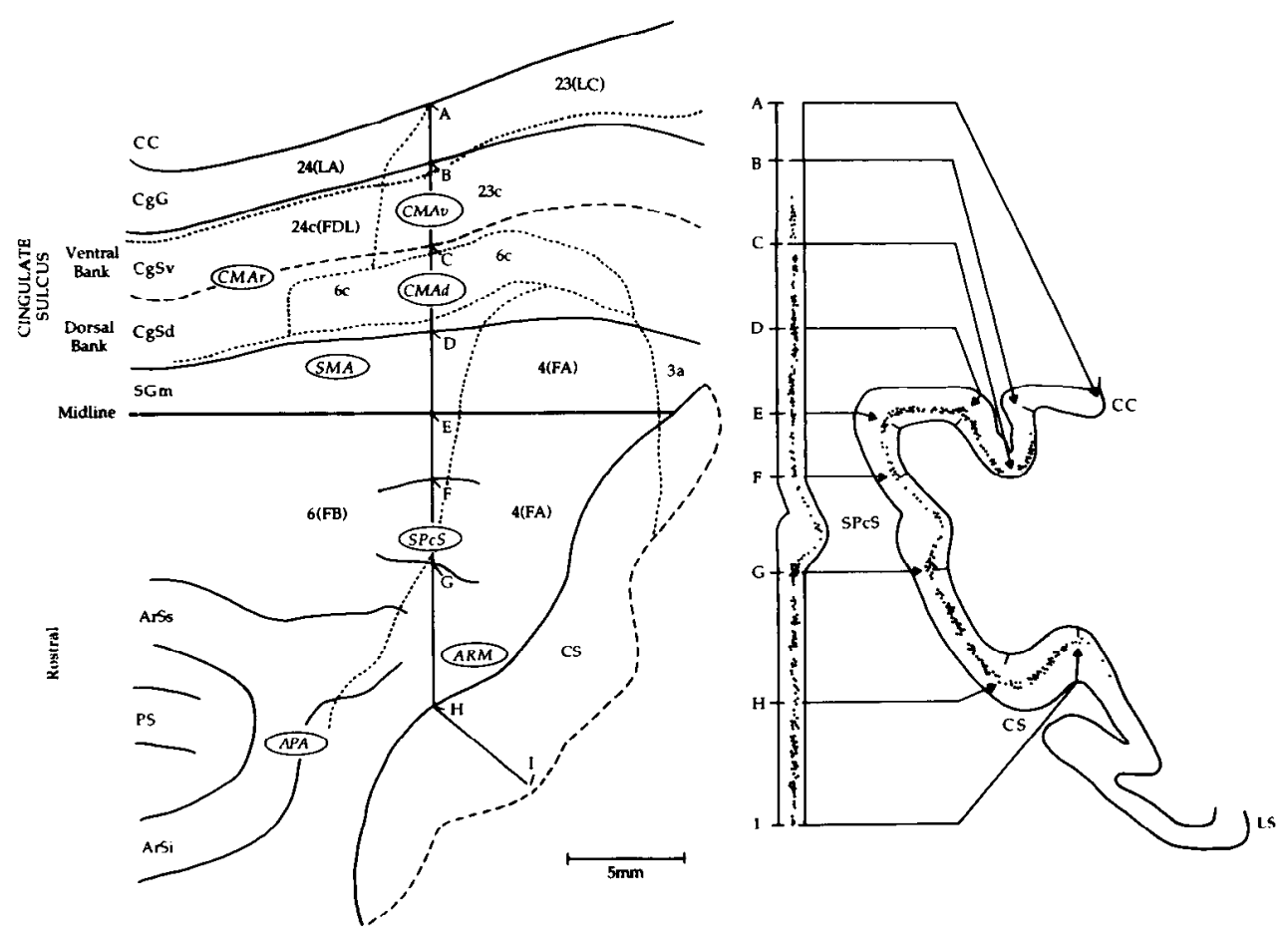

Figure 1. Schematic diagram of motor areas in frontal lobe. Individual coronal sections of cortex (far right) were straightened (middle) to form a flattened reconstruction (left) of the frontal lobe. The vertical line on the reconstruction indicates the level of the section displayed at the right. Each section was aligned on the junction of the medial wall with the lateral surface (i.e., the midline). Capital letters on the middle panel and on the vertical line in the reconstruction indicate the major points of unfolding (e.g., sulcal boundaries). The anterior bank of the central sulcus was projected perpendicular to the mean angle of the sulcus with respect to the midline. Note that the inferior and superior arcuate sulcus $(\mathrm{ArSi}$, $\mathrm{ArSs})$ and the $S P C S$ were not flattened. Cytoarchitectonic regions are identified both by numbers (Brodmann, 1905) and by letters in parentheses (von Bonin and Bailey, 1947). Dotted lines indicate borders between these cytoarchitectonic regions. A dashed line indicates the fundus of each unfolded sulcus. The centers of the different cortical motor areas in the frontal lobe are indicated by the circled lettering. APA, arcuate premotor area; $A R M$, arm representation of the primary motor cortex; $C C$, corpus callosum; $C g G$, cingulate gyrus; $C g S d$, cingulate sulcus, dorsal bank; $C g S v$, cingulate sulcus, ventral bank; $C M A d$, caudal cingulate motor area, dorsal bank; $C M A v$, caudal cingulate motor area, ventral bank; $C M A r$, rostral cingulate motor area; $C S$, central sulcus; $L S$, lateral sulcus; $P S$, principal sulcus; $S G m$, superior frontal gyrus, medial wall; $S M A$, supplementary motor area.

for a short distance onto the dorsal bank of the cingulate sulcus. Some isolated giant pyramidal cells are found in the caudal portion of area 6 on the medial wall. This feature may have led Barbas and Pandya (1987) to split area 6 on the superior frontal gyrus into a caudal area $6 \mathrm{DC}$ and a rostral area MII.

\section{Cingulate gyrus}

The cytoarchitecture of the cingulate gyrus differs substantially from that of the superior frontal gyrus. For example, the cortex on the cingulate gyrus is the thinnest on the medial wall (cf. Fig. $2 B, C$ with $A, D-F)$. The cingulate gyrus has 2 major subdivisions in the region we examined: area 23 (LC in von Bonin and Bailey, 1947) and area 24 (LA in von Bonin and Bailey, 1947; Figs. 1, $2 B, E$; for a recent review, see Vogt et al., 1987). Classically, the presence of a distinct granular layer IV in area 23 has been used to distinguish it from area 24 (Fig. $2 B, C$; e.g., von Bonin and Bailey, 1947; Vogt et al., 1987). The border between these 2 areas is found several millimeters rostral to the level of the border between areas 4 and 6 .

\section{Cingulate sulcus}

Areas 23 and 24 have been divided into a number of subfields that extend into the cingulate sulcus (e.g., Vogt et al., 1987). Rostrally, area $24 \mathrm{c}$ is found largely in the ventral bank of the sulcus. This subfield extends onto the dorsal bank of the sulcus at levels rostral to the genu of the arcuate sulcus (Fig. 1). Area 24c (the FDL of von Bonin and Bailey, 1947) is thicker than the cortex on the cingulate gyrus and, unlike the other subfields of area 24, contains an incipient granular layer (Fig. $2 F$ ). The presence of some relatively large pyramidal-shaped cells in layer $\mathrm{V}$ and in the deep part of layer III also distinguishes area $24 \mathrm{c}$ from the subfields of area 24 on the cingulate gyrus. The CMAr identified in a previous study lies within area $24 \mathrm{c}$ (Hutchins et al., 1988).

Caudally, area $23 \mathrm{c}$ is found largely in the ventral bank of the cingulate sulcus, beginning approximately $2-3 \mathrm{~mm}$ rostral to the area 4-6 border (Fig. 1). It extends onto the dorsal bank of the sulcus at levels caudal to the area $4-6$ border. Area $23 \mathrm{c}$ is markedly thicker than the other subfields of area 23 on the cingulate gyrus (cf. Fig. $2 E$ with $B$ ). It can be distinguished from area $24 \mathrm{c}$ because it has a larger number of granule cells in layer IV and a higher density of cells in the outer portions of layer III (cf. Fig. $2 E$ with $F$ ).

In their classic study, von Bonin and Bailey (1947) noted that caudal portions of the dorsal bank of the cingulate sulcus contained a distinct subfield, which they termed FAL. We observed a comparable region in the dorsal bank of the cingulate sulcus with cytoarchitectonic features that are transitional between area $23 \mathrm{c}$ and area 6 (Figs. 1,3). Because this region most resembles area 6 on the superior frontal gyrus, we consider it to be a subfield of that area. We have termed this region "area $6 c$ " to indicate its topographic location in the cingulate sulcus. Area $6 \mathrm{c}$ is dis- 

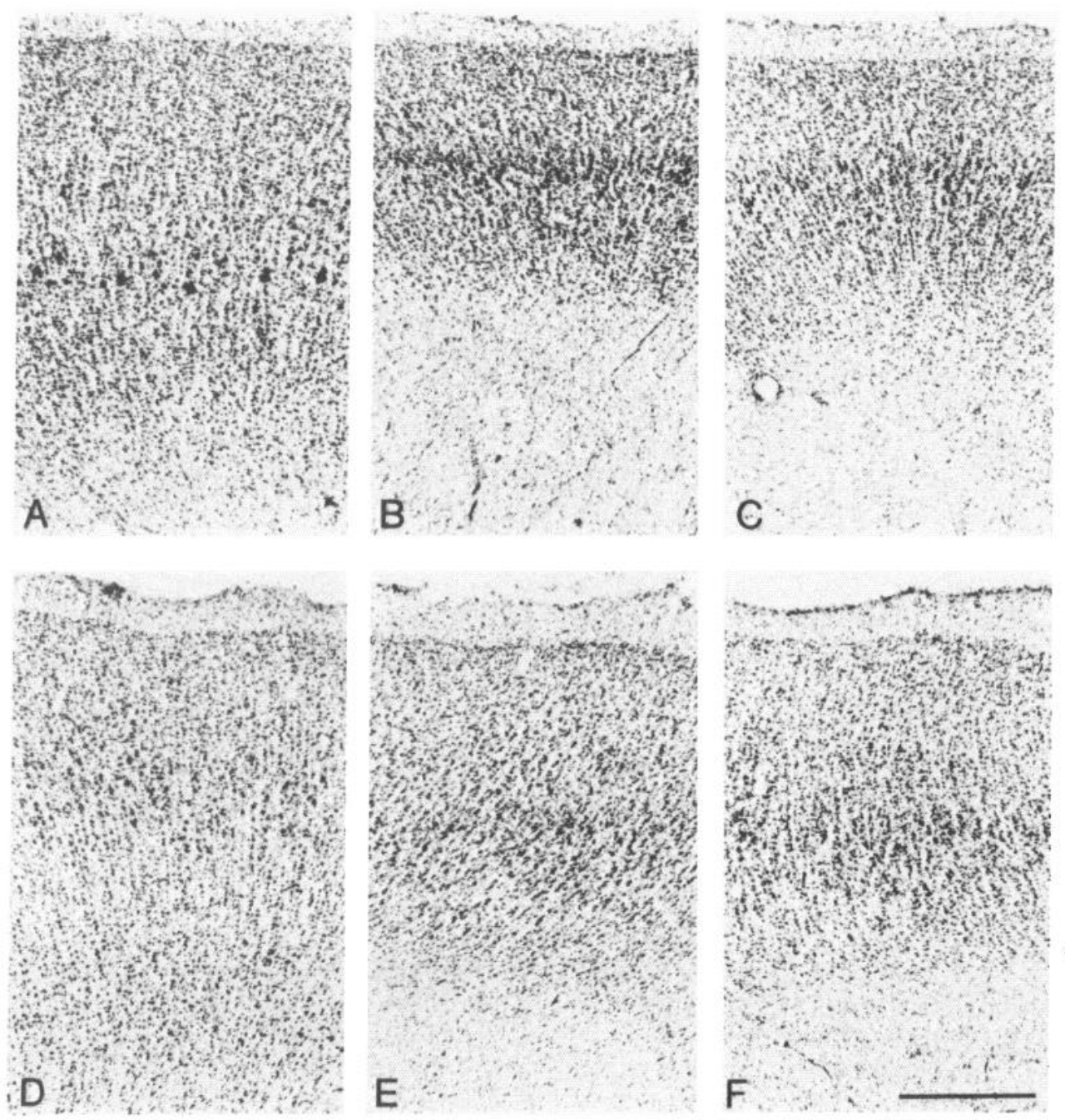

Figure 2. Cytoarchitecture of cortex on medial wall of hemisphere. These are photomicrographs of cresyl violetstained sections from a monkey that received HRP into the dorsolateral funiculus at $\mathrm{C} 2 . A$, Area 4 on the superior frontal gyrus. $B$, Area 23 on the cingulate gyrus. $C$, Area 24 on the cingulate gyrus. $D$, Area 6 on the superior frontal gyrus. $E$, Area $23 \mathrm{c}$ on the ventral bank of the cingulate sulcus, just caudal to the area 23-24 border. $F$, Area 24c on the ventral bank of the cingulate sulcus. Scale bar, $500 \mu \mathrm{m}$. tinguished from adjacent parts of areas 4 and 6 by a clear decrease in cortical thickness, particularly in layer VI (Fig. 3). It is distinguished from area $23 \mathrm{c}$ by the absence of a granular layer IV and the presence of a few large, pyramidal-shaped cells in layer V. Also, layers V and VI in area $6 \mathrm{c}$ are thicker than in area 23c. Previously, we considered the corticospinal projections from area $6 \mathrm{c}$ and area $23 \mathrm{c}$ to lie within the CMAc (Hutchins et al., 1988). However, the cytoarchitectonic differences between these subfields, the detailed patterns of their corticospinal projections (see below), and the results of recent connectional studies (Holsapple and Strick, 1989) have led us to consider that portion of the CMAc in area $6 c$ on the dorsal bank of the cingulate sulcus (CMAd) as separate from that portion of the CMAc in area $23 \mathrm{c}$ on the ventral bank (CMAv).

\section{Location and extent of injection sites}

We used 2 experimental approaches to determine the origin of corticospinal projections to cervical segments of the spinal cord. In one series of experiments $(n=2)$, we transected the dorsolateral funiculus at $\mathrm{C} 2$ and inserted HRP crystals into the lesion site. In the second series $(n=2)$, we injected WGA-HRP into the gray matter throughout multiple segments of the cervical spinal cord. We will present detailed descriptions of the injection sites and the cortical labeling of 1 representative animal from each series.

\section{HRP into the dorsolateral funiculus}

In order to label all the corticospinal fibers that travel in the lateral component of this tract, we placed HRP into the dorsolateral funiculus at $\mathrm{C} 2$. Sections of spinal cord rostral to the site of HRP placement demonstrated that axons were labeled throughout the full cross-sectional area of the ipsilateral lateral funiculus (Fig. 4B). In addition, we observed large numbers of labeled axons ipsilaterally in the lateral part of the ventral funiculus and in the lateral part of the dorsal column. Only small numbers of labeled axons were found in the medial part of the ventral funiculus. The only labeled axons found contralaterally were seen ventromedially in the dorsal column. Thus, the injection site completely covered the lateral component of the corticospinal tract, but spared other components of the corticospinal system, including those that travel in ipsilateral funiculi (e.g., Kuypers, 1960; Liu and Chambers, 1964; Kuypers and Brinkman, 1970; Ralston and Ralston, 1985).

\section{WGA-HRP into the gray matter}

In order to label those corticospinal neurons involved in the control of arm movements, we injected WGA-HRP into multiple segments of the cervical spinal cord. The injection needle was angled to pass through the dorsal columns (see Materials and Methods) to avoid damage and/or spread of the tracer to 
Figure 3. Cytoarchitecture in cingulate sulcus. The level of this section is close to the vertical line drawn on the flattened reconstruction in Figure 1. Arrows indicate the boundaries of areas $6 c$ and $23 \mathrm{c}$. Scale bar, $1.0 \mathrm{~mm}$.

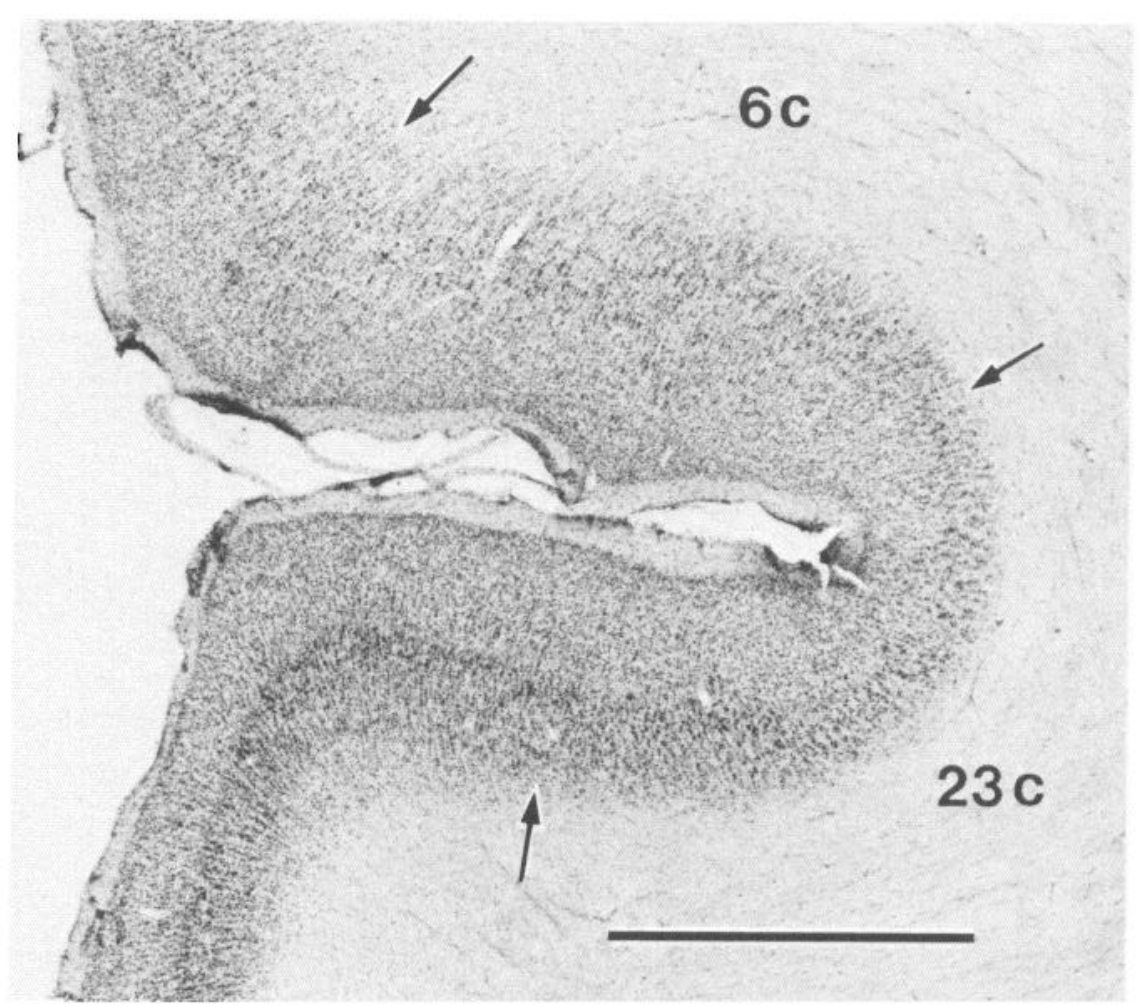

the lateral funiculus. In fact, on sections caudal to the injection site, we found labeled axons largely confined to the region of the lateral funiculus that was adjacent to the gray matter of the spinal cord (Fig. 4A). This is the region where propriospinal pathways are known to travel (Kuypers, 1981). This observation suggests that tracer uptake was limited to the gray matter within the injection site.

We defined the injection site in gray matter as the zone of staining that contained the densest reaction product (Mesulam, 1982). In this zone, labeled cells could not be distinguished from the staining of the neuropil. In the representative animal from this series, the injection site involved the entire dorsal horn, intermediate zone, and ventral horn from the sixth cervical segment (C6) to the first thoracic segment (T1; Fig. 5). Caudally, the injection site extended into the intermediate zone and ventral horn of the second thoracic segment (T2). Rostrally, the injection site partially involved the ventral horn and intermediate zone of the fourth and fifth cervical segments $(\mathrm{C} 4, \mathrm{C} 5)$.

\section{Distribution of corticospinal neurons in the frontal lobe}

\section{HRP into the dorsolateral funiculus at $\mathrm{C} 2$}

Multiple cortical areas in the frontal lobe contained substantial numbers of labeled neurons after HRP placement into the dorsolateral funiculus at $\mathrm{C} 2$ (Figs. 6, 7). Large numbers of labeled neurons were found in regions of area 4 located on the precentral gyrus, the anterior bank of the central sulcus, and the medial wall of the hemisphere. These parts of area 4 contain the forelimb and hindlimb representations of the primary motor cortex (e.g., Woolsey et al., 1952; Kwan et al., 1978; Wise and Tanji, 1981; Sessle and Wiesendanger, 1982).

In general, the number of labeled neurons on the lateral surface of the hemisphere gradually declined after the area 4-6 border. Only a few labeled neurons were found in the rostral part of area 6 located medial to the superior limb of the arcuate sulcus and rostral to its genu (Figs. 6; 7, sections 176, 217, 245). However, there were 2 parts of area 6 on the lateral surface of the hemisphere that contained substantial numbers of labeled neurons. One of these was located in the caudal strip of area 6, which includes the shallow SPcS (Figs. 6; 7, sections 329, 357). The other was located in a more lateral part of area 6 (Figs. 6; 7 , sections $217,245,289$ ). Labeled neurons there were located largely in the caudal bank of the inferior limb of the arcuate sulcus and continued medially to include regions in and around the spur of this sulcus. This part of area 6 corresponds to the arm representation of the APA (Matsumura and Kubota, 1979; Muakkassa and Strick, 1979; Godschalk et al., 1984; Strick, 1985; Leichnetz, 1986; Matelli et al., 1986; Ghosh et al., 1987; Martino and Strick, 1987).

Substantial numbers of labeled neurons also were found in several cytoarchitectonic regions on the medial wall of the hemisphere. Labeled neurons on the superior frontal gyrus were found in the hindlimb representation of area 4 and in the regions of area 6 rostral to it (Figs. 6; 7, sections 217-357, area 4 on sections $385-481$ ). These neurons were found as far rostrally as $2 \mathrm{~mm}$ beyond the genu of the arcuate sulcus. The part of area 6 on the superior frontal gyrus that contained labeled neurons coincides with the fore- and hindlimb representations of the SMA (e.g., Woolsey et al., 1952; Brinkman and Porter, 1979; Muakkassa and Strick, 1979; Wise and Tanji, 1981; Macpherson et al., 1982; Mitz and Wise, 1987; Hutchins et al., 1988).

Labeled neurons were found throughout a considerable rostrocaudal extent of both the dorsal and ventral banks of the cingulate sulcus (Fig. 6,7). These neurons were located as far rostrally as $4 \mathrm{~mm}$ anterior to the genu of the arcuate sulcus and as far caudally as the end of the sulcus. Sites containing labeled neurons included all of area 6c (CMAd) and substantial portions 

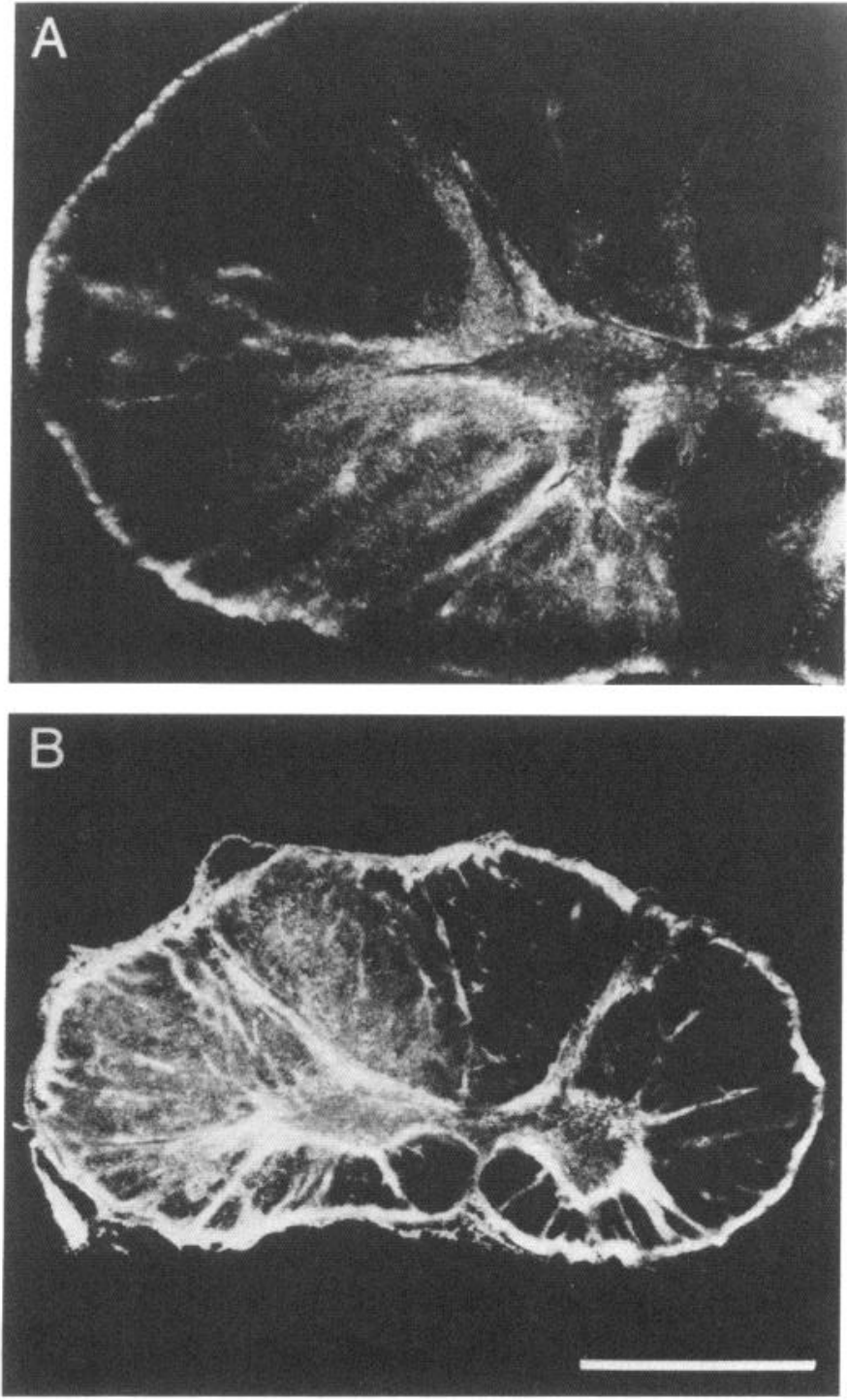

Figure 4. Spinal cord injection sites. These photomicrographs were taken under dark-field/polarized light illumination. $A$, Cross-section of the spinal cord caudal to an injection of WGA-HRP into the gray matter throughout C4-T2. Labeled axons caudal to the inject site were confined to the white matter immediately adjacent the gray matter. No labeled axons were visible in the more lateral regions of the dorsolateral funiculus. $B$, Cross-section of the spinal cord $1.5 \mathrm{~mm}$ rostral to the placement of HRP crystals into the dorsolateral funiculus at $\mathrm{C} 2$. Most of the HRP reaction product was confined to axons in the ipsilateral (left) dorsal columns, dorsolateral funiculus, and ventrolateral funiculus. The location of the labeled axons corresponds with the extent of the lesion observed in more caudal sections. Scale bar: $930 \mu \mathrm{m}$ for $A ; 2.0 \mathrm{~mm}$ for $B$.

of areas 23c (CMAv) and 24c (CMAr). Labeled neurons were rarely found on the cingulate gyrus. Thus, in addition to the primary motor cortex, corticospinal neurons were found in 6 different regions of the frontal lobe, which we have identified as premotor areas: the APA, the portion of area 6 around the SPcS, the SMA, the CMAr, the CMAd, and the CMAv.

As in prior studies (e.g., Jones and Wise, 1977; Murray and Coulter, 1981), we found that, on single sections through each motor area, labeled cortical neurons were arranged in irregular clusters (Figs. 6-9). These clusters were present after tracer injections into either the dorsolateral funiculus or the gray matter
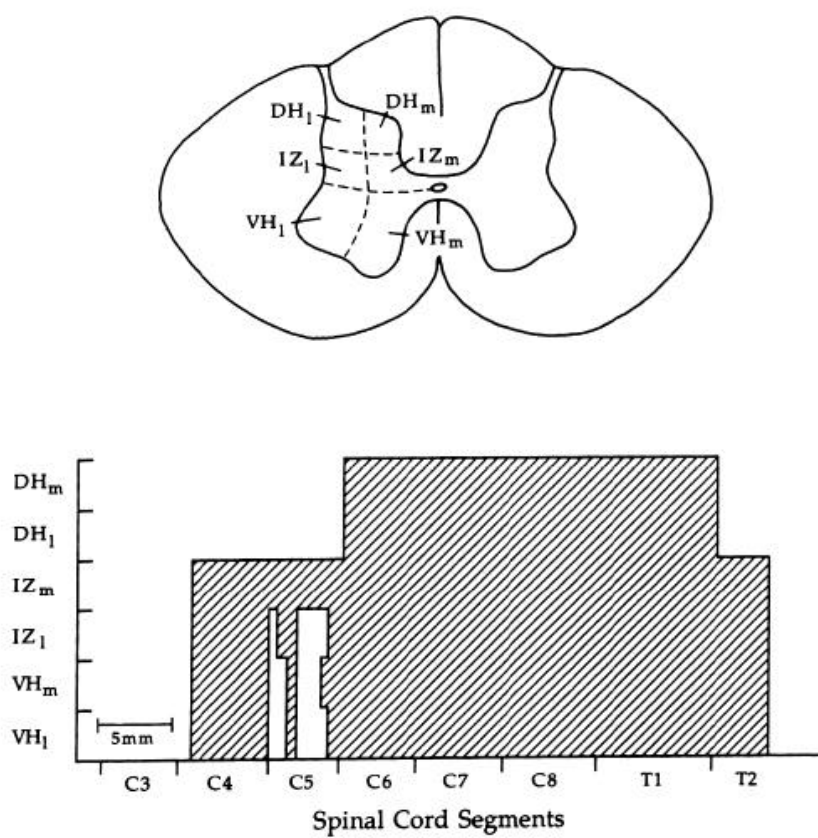

Figure 5. Diagrammatic representation of WGA-HRP injection site. Top, Drawing of a cross-section through C8 indicating the 6 zones of gray matter that were evaluated for reaction product. The zones included the medial $(m)$ and lateral $(l)$ part of the dorsal horn $(D H)$, intermediate zone $(I Z)$, and ventral horn $(V H)$. Bottom, The histogram indicates the presence of reaction product within each of the 6 zones throughout the cervical enlargement.

of the spinal cord. However, "stripes" or "bands" of labeled neurons were not clearly evident in our surface reconstructions (Figs. 6, 8) or maps of neuron density (see Figs. 11, 12). Future studies will need to employ additional technical and analytic procedures (e.g., Johnson et al., 1989) to define the arrangement of these clusters of corticospinal neurons in each of the premotor areas.

\section{WGA-HRP into cervical segments}

Injections of WGA-HRP into the gray matter of the cervical spinal cord (C4-T2) resulted in neuronal labeling in parts of the same cytoarchitectonic areas as were labeled following funicular deposits of HRP at C2 (cf. Figs. 6, 8). Indeed, after injections into the cervical cord, we found substantial numbers of labeled neurons in the primary motor cortex and in each of the 6 premotor areas in the frontal lobe. However, these neurons were topographically restricted to specific parts of each region. This is in striking contrast to the more uniform distribution of labeled neurons observed after placement of HRP into the lateral funiculus.

Although their distribution was restricted, the number of corticospinal neurons found within each labeled region was greater in animals with injections of WGA-HRP than in those with the deposits of HRP. This difference was particularly evident in the numbers of labeled neurons in the arcuate sulcus (cf. Fig. 7, section 217, with Fig. 9, section 209). It is unclear whether this quantitative difference is due to (1) the tracer employed (WGAHRP vs. HRP; e.g., Gonatas et al., 1979; Mesulam, 1982), (2) the site of injection and parameters of uptake (cut axons in the funiculus vs. gray matter injection sites; e.g., Brushart and Mesulam, 1980; Mesulam, 1982), or (3) variability among animals 
Figure 6. Map of corticospinal neurons in frontal lobe. HRP was injected into the contralateral dorsolateral funiculus at $\mathrm{C} 2$ (shown in Fig. $4 B$ ). Each labcled cell is represented by a dot. Every other $50-\mu \mathrm{m}$ section was used to construct this map. See Materials and Methods and Figure 1 for the reconstruction procedures and abbreviations. The numbered scale shows the location of sections illustrated in Figure 7.

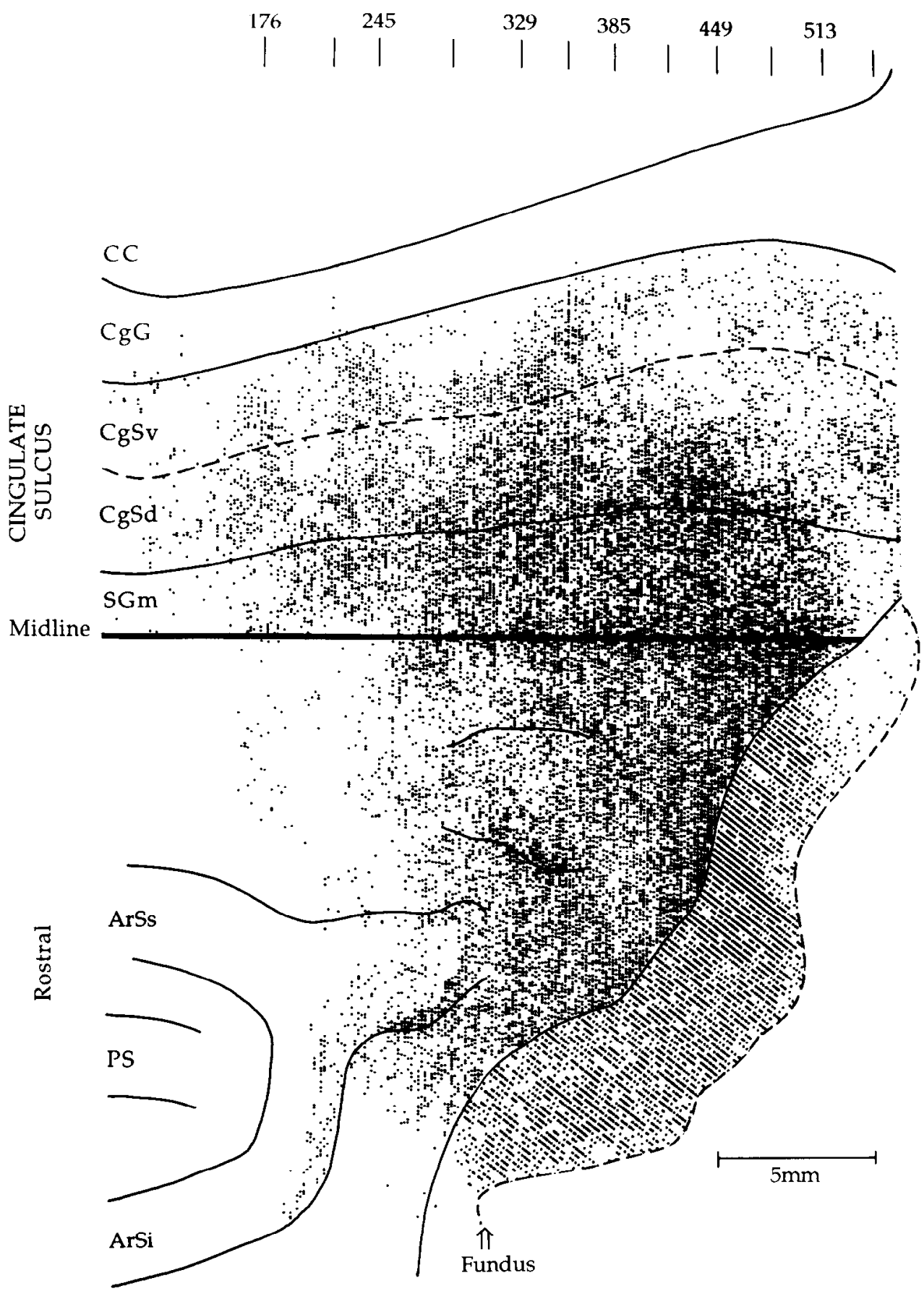

CENTRAL SULCUS

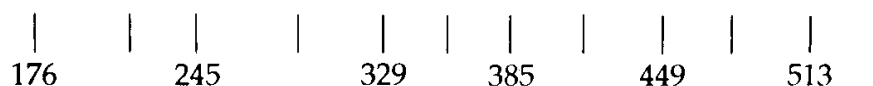

in the number of pyramidal-tract axons (c.g., Russell and DeMeyer, 1961).

Within area 4 , labeled neurons were confined to a region that began medially at the level of the SPcS and extended slightly lateral to the spur of the arcuate sulcus. Large numbers of labeled neurons were found on both the precentral gyrus and the anterior bank of the central sulcus. This labeled region corresponds to the forelimb representation in the primary motor cortex (e.g., Woolsey et al., 1952; Kwan et al., 1978; Sessle and Wiesen- danger, 1982). No corticospinal neurons were found in the face representation of area 4 , and very few were seen in the hindlimb representation. The absence of labeling in the hindlimb region of the primary motor cortex provides additional evidence for our claims that (1) corticospinal axons passing in the funiculi to lower levels of the cord were not involved by our injections into gray matter, and (2) tracer uptake in these animals was limited to cervical segments of the spinal cord.

Another group of labeled neurons was found in the region of 

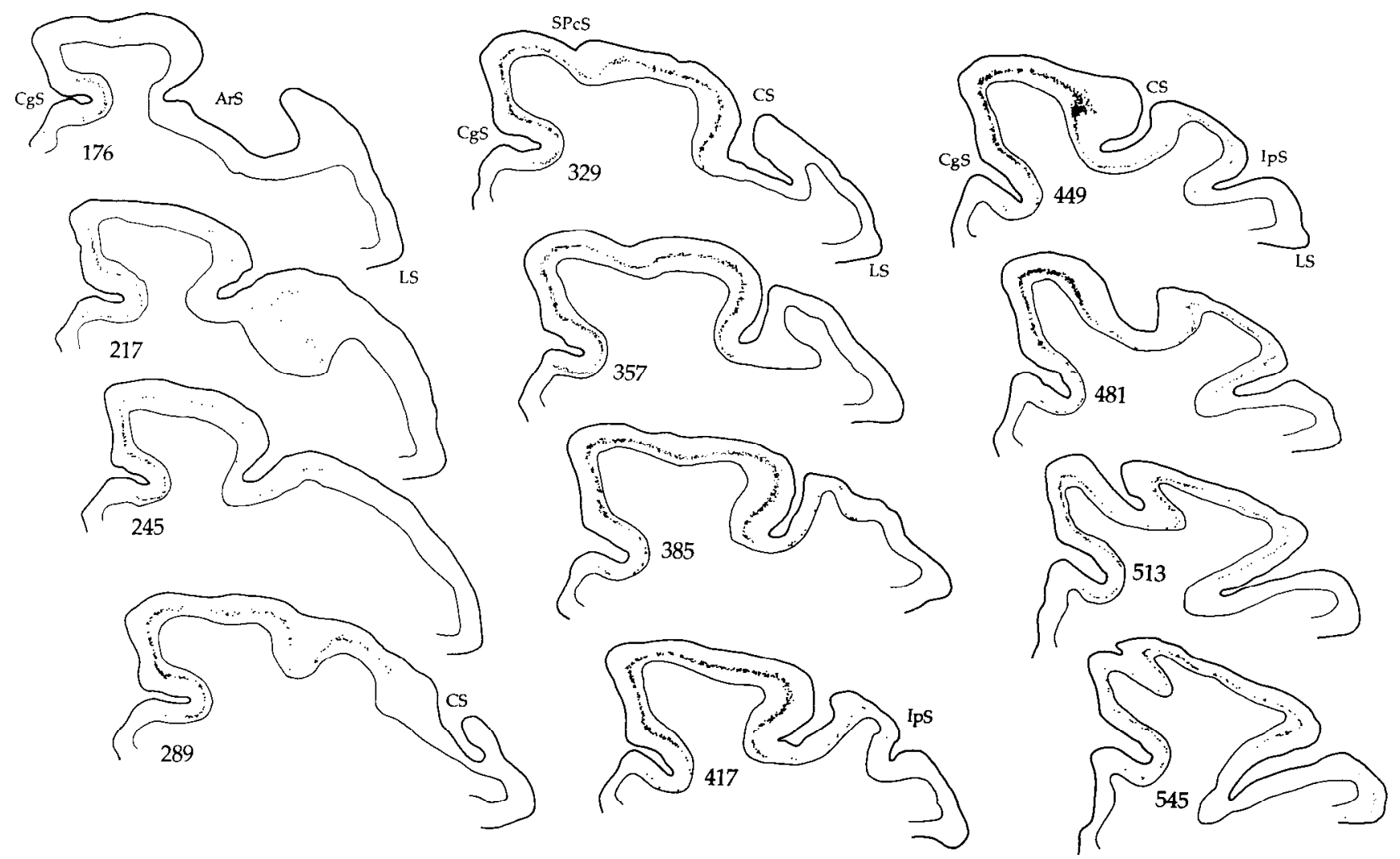

Figure 7. Plots of corticospinal neurons found on coronal sections through frontal lobe. The coronal sections are from the animal illustrated in Figure 6; the location of each section is indicated by the numbered scale in that figure. $C g S$, cingulate sulcus; $I p S$, intraparietal sulcus; other abbreviations are as in Figure 1.

area 6 that surrounds the lateral edge of the SPcS (Figs. 8; 9 , sections $329,357,381$ ). This group of labeled neurons merged caudally and laterally, with the main concentration of labeled neurons in the primary motor cortex. The labeled neurons in and around the SPcS overlap the arm representation of the premotor area located in this region of the cortex (e.g., Muakkassa and Strick, 1979; Godschalk et al., 1984; Kurata et al., 1985; Kurata, 1989). A small cluster of labeled neurons was located immediately caudal to the medial lip of the SPcS (Fig. 8). Neurons were labeled at this location only when gray matter injections included lower segments (C7-T1) of cervical spinal cord (Dum and Strick, 1989; He et al., 1989).

A distinct patch of labeled neurons also was found more laterally in area 6 . These labeled neurons were buried in the caudal bank of the arcuate sulcus, particularly its inferior limb (Figs. $8 ; 9$, section $209 ; 10$ ). This area of labeling was isolated from the main concentration of labeled neurons in area 4 by a sparsely labeled region along the arcuate spur (Fig. 8; see also Nudo and Masterton, 1990). The topographic distribution of these neurons was best visualized on 3-D reconstructions of the caudal bank of the arcuate sulcus (Fig. 10). Such reconstructions demonstrated that corticospinal neurons tended to shift from a superficial position in the sulcus at caudomedial levels to the depths of the sulcus at more rostrolateral levels. This region of corticospinal neurons corresponds to the arm representation of the APA (e.g., Matsumura and Kubota, 1979; Muakkassa and Strick, 1979; Godschalk et al., 1984, 1985; Martino and Strick, 1987; Gentilucci et al., 1988).
On the medial wall of the hemisphere, restricted portions of the superior frontal gyrus and the banks of the cingulate sulcus contained concentrations of labeled neurons. These labeled areas were separated by regions that either lacked or had few labeled neurons. This pattern of labeling is in marked contrast to the more uniform distribution of labeled neurons found on the medial wall after funicular injections of tracer (cf. Figs. 6, $8)$.

On the superior frontal gyrus, most corticospinal neurons were located in the region of area 6 that started at the level of the genu of the arcuate sulcus and extended caudally for approximately $6 \mathrm{~mm}$. The largest number of these labeled neurons was found ventrally on the gyrus. Very few labeled neurons were observed in regions that extend either rostral to the genu of the arcuate sulcus or dorsally onto the lateral surface. Thus, these corticospinal neurons were located largely in a region that coincides with the arm representation of the SMA (e.g., Penfield and Welch, 1951; Woolsey et al., 1952; Brinkman and Porter, 1979; Muakkassa and Strick, 1979; Macpherson et al., 1982; Mitz and Wise, 1987; Hutchins et al., 1988).

Rostrally, the dorsal and ventral banks of the cingulate sulcus contained scattered clusters of labeled neurons (Figs. 8; 9, sections 133,173$)$. These corticospinal neurons were located in the region of area $24 \mathrm{c}$ that was rostral to the genu of the arcuate sulcus (Figs. 8; 9, sections 209, 237). This region of area 24c corresponds to the arm representation of the CMAr (Hutchins et al., 1988; Dum and Strick, 1989; He et al., 1989). The region of the dorsal and ventral banks of the cingulate sulcus that lies 
Figure 8. Map of corticospinal neurons in frontal lobe projecting to $\mathrm{C} 4$ T2. WGA-HRP was injected into the contralateral gray matter throughout C4-T2. Every fourth section was used to construct this map. See Figures 1 and 6 for conventions and methods of reconstruction.

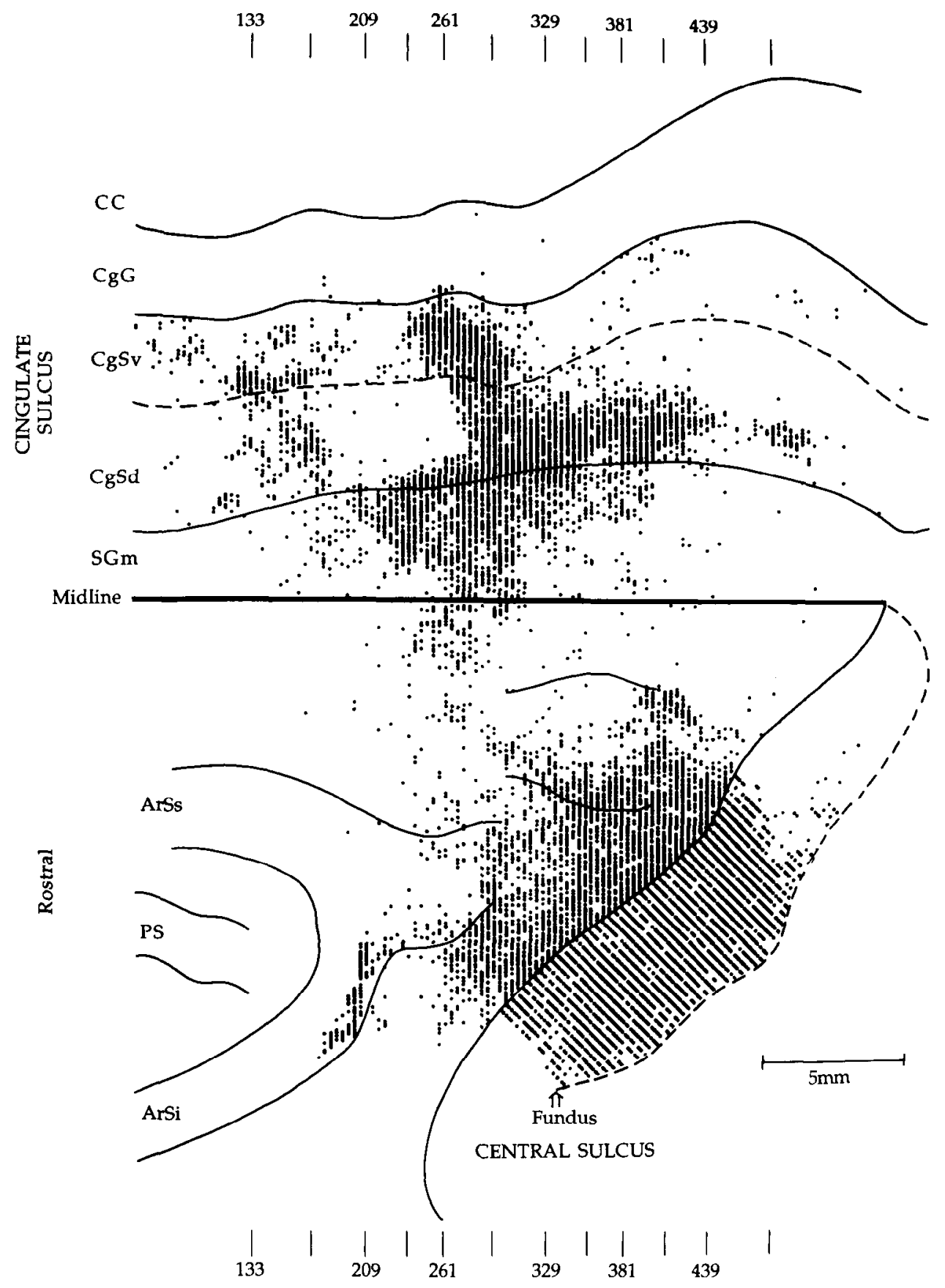

$293,329)$. These neurons were located in area 23c. In a subsequent section, we will present evidence that the region with corticospinal neurons in area $6 \mathrm{c}$ corresponds to the arm representation of the CMAd and the region in area $23 \mathrm{c}$ corresponds to the arm representation of the CMAv (see also Hutchins et al., 1988; Dum and Strick, 1989, 1990; He et al., 1989).

Using the surface maps, we measured the area of each cortical region that contained labeled neurons after injections of tracer into cervical segments of the spinal cord (Table 1). The total area of cortex in the frontal lobe that contained corticospinal neurons measured $226 \mathrm{~mm}^{2}$. Only $37 \%$ of this area was located in the arm area of the primary motor cortex. The next largest area containing corticospinal neurons was the arm area of the SMA (19\%). Smaller amounts of the cortex were allotted to the 


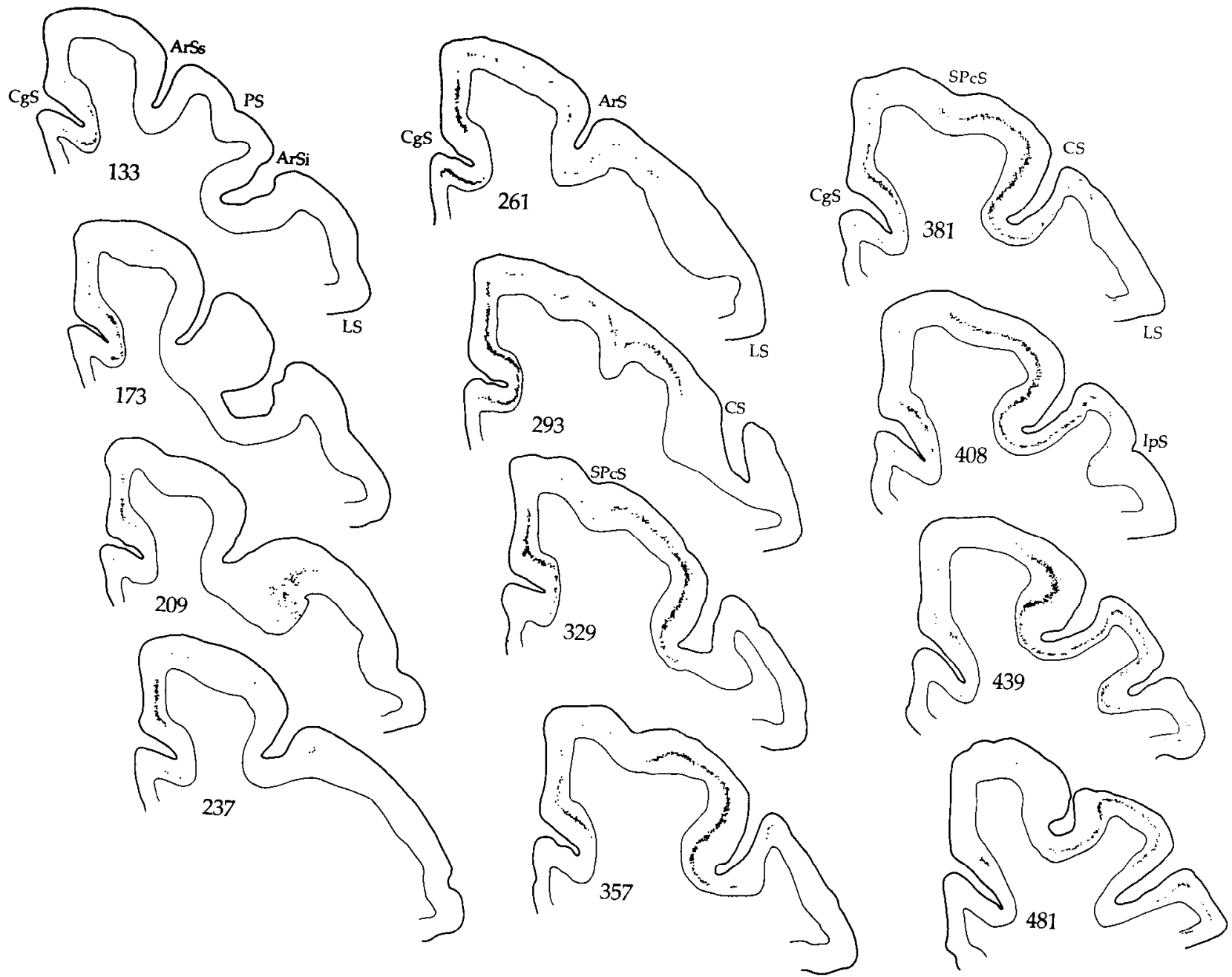

Figure 9. Plots of corticospinal neurons projecting to C4-T2 found on coronal sections through frontal lobe. The coronal sections are from the animal illustrated in Figure 8; the location of each section is indicated by the numbered scale in that figure. Abbreviations are as in Figures 1 and 7.

arm representations of the other premotor areas (CMAr, $11 \%$; CMAd, 10\%; SPcS, 9\%; APA, 8\%; CMAv, 6\%).

\section{Density of corticospinal neurons in the motor areas}

The surface maps of labeled neurons provide an accurate representation of the topographic distribution of corticospinal neurons in the frontal lobe. However, when every labeled neuron is plotted, these maps suffer from a tendency to distort the apparent density of corticospinal neurons at some sites. For example, the plot of labeled neurons saturated into a solid line at sites where there was a moderate to high density of corticospinal neurons. To overcome this and other shortcomings, we constructed surface maps that displayed the density of labeled neurons (for further description, see Figs. 11, 12 and Materials and Methods).

These maps demonstrated that high-density bins (i.e., bins in which the number of labeled neurons was in the upper $10 \%$ of the total sample of $200-\mu \mathrm{m}$ bins) were found in each of the 6 premotor areas, as well as in the primary motor cortex (Figs. 11,12 ). High-density bins were scattered throughout the primary motor cortex. However, they were most frequently found on the edge and in the anterior bank of the central sulcus (Fig. 12). At more rostral portions of the primary motor cortex, there was a decline in the density of labeled neurons. Then, the number of high-density bins increased in the premotor area at the lateral edge of the SPcS.

On the medial wall of the hemisphere, high-density bins were particularly concentrated in the CMAd and CMAv (Fig. 11). Furthermore, a trough in density clearly separated the mass of high-density bins in the CMAd (in area 6c) from those in the CMAv (in area 23c). Within the SMA, high-density bins were most concentrated ventrally near the edge of the cingulate sulcus.

In total, we found 450 high-density bins in the frontal lobe of the animal illustrated in Figures 11 and 12. Fifty-three percent of these were located in the arm representation of the primary motor cortex. The next largest groups of high-density bins were located in the arm area of the SMA (19\%) and in the arm area of the CMAd $(15 \%)$. Smaller groups of high-density bins were found in the arm representations of the other premotor areas (CMAv, 7\%; SPcS, 5\%; CMAr, <1\%; APA, <1\%).

We determined the distribution of density bins for each premotor area and compared them with the distribution of density bins for the primary motor cortex (Fig. 13). The distributions for 2 premotor areas (CMAd and CMAv) were remarkably similar to that of the primary motor cortex $\left(\chi^{2}, p=>0.1\right)$. In contrast, the distributions for the APA and CMAr were highly 
Figure 10. Corticospinal neurons in arcuate sulcus. This is a $3-\mathrm{D}$ reconstruction of the caudal bank of the arcuate sulcus from the animal illustrated in Figures 8 and 9 (WGA-HRP into C4$\mathrm{T} 2$ ). The labeled neurons (dots) from every other section are plotted along with the surface outline of every eighth section. The area reconstructed is indicated by the dashed lines on the diagram of the brain surface at the lower left. ArSpur, spur of the arcurate sulcus.

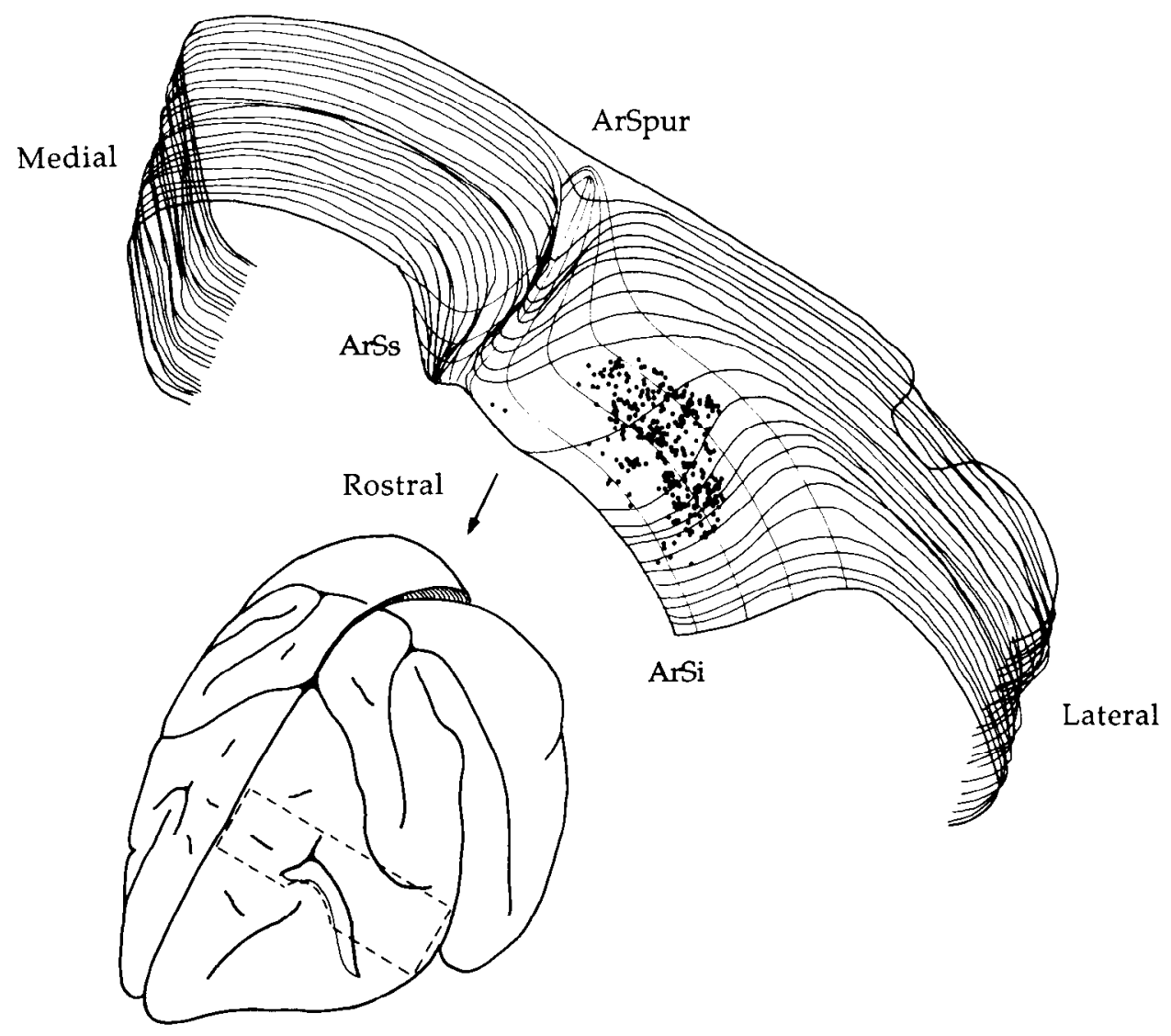

skewed toward low-density bins $(p=<0.0001)$. The distributions in the remaining premotor areas were only slightly skewed toward low-density bins $(p=<0.05)$.

\section{Number of corticospinal neurons in the motor areas}

We counted the number of labeled neurons found on every fourth section through each of the motor areas and multiplied this number by 4 to get the total number of corticospinal neurons in each area. In the animal illustrated (Figs. 8-12; WGA-HRP injections into gray matter), we found 42,912 corticospinal neurons in the frontal lobe that projected to cervical segments of the spinal cord. Less than $50 \%$ of these neurons were located in the arm area of the primary motor cortex (Table 1). Within the premotor areas, the largest numbers of corticospinal neurons were located within the arm area of the SMA $(18.5 \%$ of the total) and in the arm area of the CMAd (10.5\%). Smaller numbers of corticospinal neurons were located in the arm representations of the other premotor areas. More corticospinal neurons $(n=71,128)$ were labeled in another animal with a more extensive injection of WGA-HRP into cervical segments of the spinal cord (from $\mathrm{C} 2$ to $\mathrm{T} 1$ ). As in the animal illustrated, less than $50 \%$ of these neurons were located in the arm area of the primary motor cortex (Table 1). Also, the arm area of the SMA contained more corticospinal neurons than any of the other premotor areas $(12.2 \%)$. However, in this animal, larger percentages of corticospinal neurons were located in the arm areas of the APA and SPcS.

\section{Size of corticospinal neurons in the motor areas}

We determined the mean diameter of corticospinal neurons in each of the motor areas in the frontal lobe. Because other studies have shown that the size of corticospinal neurons varies with their level of segmental termination (e.g., Biber et al., 1978; Murray and Coulter, 1981; Toyoshima and Sakai, 1982), our analysis was restricted to an animal with tracer injections into cervical segments of the spinal cord (Figs. 8-12). The distribution of soma diameters for the total sample of corticospinal neurons in the frontal lobe ranged from 9 to $31 \mu \mathrm{m}$ (Fig. 14). For comparative purposes, we have defined "large neurons" as those with soma diameters greater than $19 \mu \mathrm{m}$. Using this definition, we found large neurons in all of the motor areas of the frontal lobe except the CMAr and the CMAv (see Fig. 16). The greatest number of large corticospinal neurons was located in the arm area of the primary motor cortex. Thirty-one percent of the corticospinal neurons in this region were large, and these neurons represented $79 \%$ of the total number of large corticospinal neurons. Although a sizeable portion of the corticospinal neurons in the arm areas of the $\Lambda P \Lambda^{\prime}$ and the SPcS were large (APA, 20\%; SPcS, 21\%), together these regions contained only $12 \%$ of all the large corticospinal neurons in the frontal lobe. A

I The somas of most of the neurons in the APA were not aligned parallel to the plane of sectioning. Consequently, the measurements of their soma diameter underestimated their size. 
relatively small percentage of the corticospinal neurons in the arm areas of the SMA and CMAd were large (6\% in each area). Within the arm area of the SMA, these neurons were concentrated caudally and ventrally on the superior frontal gyrus. Finally, corticospinal neurons in the arm areas of the CMAr and the CMAv were significantly smaller (Figs. 15, 16) than those found in the other motor areas of the frontal lobe (Mann-Whitney $U$ test, $p=<0.001$ ).

\section{Projections from the medial wall to the arm area of the primary motor cortex}

A final goal of our experiments was to compare the location of those regions on the medial wall that project to cervical segments of the spinal cord with the premotor areas in this region of the cortex. A similar comparison for the arm area of the APA was the topic of a previous report (Martino and Strick, 1987). We defined the location of the arm representations in the premotor areas by making multiple injections of WGA-HRP into the arm area of primary motor cortex $(n=2$; see Materials and Methods, Fig. 17). The results from 1 representative animal will be presented in detail. The injection site in this animal was defined as the zone of dense reaction product where labeled neurons could not be distinguished from the staining of neuropil (Mesulam, 1982; the dark line on the inset of Fig. 17). Based on this definition, the injection site involved a large portion of the arm area of the primary motor cortex on the precentral gyrus and in the anterior bank of the central sulcus. The lateral edge of the injection site may have encroached upon the face representation in the primary motor cortex (e.g., Woolsey et al., 1952; McGuinness et al., 1980; Huang et al., 1988). However, the location of the injection site is comparable to the location of labeled neurons found in the primary motor cortex of other animals after tracer injections into cervical segments of the spinal cord (Fig. 8; see also Martino and Strick, 1987; Dum and Strick, 1989).

Large numbers of corticocortical neurons were found in the SMA, CMAr, CMAd, and CMAv (Figs. 17, 18). In fact, the map of labeled neurons on the medial wall of the hemisphere in this animal was remarkably similar to those of animals with tracer injections into cervical segments of the spinal cord (cf. Figs. 17,18 with 8,11 ). Both corticocortical and corticospinal neurons within the SMA were densest at a level caudal to the genu of the arcuate sulcus (Fig. 18). In both instances, the arm representations in the CMAd and CMAv were larger and contained more efferent neurons than the arm representation in the CMAr. Also, corticocortical neurons, like corticospinal neurons, were absent from a region of the dorsal bank of the cingulate sulcus located between the CMAr and CMAd.

Even some of the fine details in the distribution of corticocortical neurons matched the distribution of corticospinal neurons. For example, there was a trough in the density of corticocortical neurons that separated the labeled neurons in the arm area of the CMAd from those in the CMAv (Fig. 18). This trough fell on the border between area $23 \mathrm{c}$ and area $6 \mathrm{c}$. A similar trough was present in the density maps of corticospinal neurons (e.g., Fig. 11). ${ }^{2}$ Finally, a small cluster of corticocortical neurons was found on the dorsal bank of the cingulate sulcus just caudal to

\footnotetext{
${ }^{2}$ Another trough in the density of corticocortical neurons was present on the superior frontal gyrus (Fig. 18). However, a comparable trough was not clcarly apparent at this location in the density maps of corticospinal neurons (e.g., Fig. 11).
}

Table 1. Distribution of corticospinal neurons among the premotor areas and the primary motor cortex

\begin{tabular}{|c|c|c|c|}
\hline & & $\begin{array}{l}\text { WGA-HRP in } \\
\text { C4-T2 }\end{array}$ & $\begin{array}{l}\text { WGA-HRP in } \\
\text { C2-T1 }\end{array}$ \\
\hline Region & $\left(\mathrm{mm}^{2}\right)$ & $\%$ & $\%$ \\
\hline
\end{tabular}

\section{Primary}

motor

\begin{tabular}{lrrrrr}
\multicolumn{1}{c}{ cortex } & 84 & 20,824 & 48.5 & 35,344 & 49.7 \\
SPcS & 20 & 3004 & 7.0 & 7032 & 9.9 \\
APA & 18 & 1716 & 4.0 & 6824 & 9.6 \\
SMA & 44 & 7952 & 18.5 & 8704 & 12.2 \\
CMAd & 22 & 4496 & 10.5 & 5664 & 8.0 \\
CMAv & 14 & 2900 & 6.8 & 4336 & 6.1 \\
CMAr & 24 & 1728 & 4.0 & 2056 & 2.9 \\
Area 6DR & $-^{a}$ & $\frac{292}{42,912}$ & $\frac{0.7}{100.0}$ & $\frac{1168}{71,128}$ & $\frac{1.6}{100.0}$
\end{tabular}

$\overline{\text { Results from } 2 \text { animals are included (WGA-HRP into C4-T2 and WGA-HRP }}$ into $(2-T 1)$.

a Area 6DR of Barbas and Pandya (1987).

${ }^{b}$ The surface area of this region was not included because it does not meet our definition of a premotor area (see Discussion).

the main group of labeled neurons in the CMAd. An analogous cluster of labeled neurons also was present in the maps of corticospinal neurons (cf. Figs. 11, 18). These observations indicate that precisely the same portions of the premotor areas that project to the arm area of the primary motor cortex also innervate the cervical segments of the spinal cord.

\section{Discussion}

\section{Identification of the premotor cortex}

As noted in the introductory remarks, we have defined the premotor cortex as those regions in the frontal lobe that project directly to the primary motor cortex. Based on this definition, we have found that the premotor cortex is composed of multiple, spatially separate areas (e.g., Muakkassa and Strick, 1979; Schell and Strick, 1984; Strick, 1985; Dum and Strick, 1990; see also Matsumura and Kubota, 1979; Godschalk et al., 1984; Leichnetz, 1986; Ghosh et al., 1987). These premotor areas are located in diverse cytoarchitectonic regions, including subfields of areas 6,23 , and 24 . The present study demonstrates that the areas comprising the premotor cortex share another important feature, namely, that each has a projection to the spinal cord. Consequently, the premotor areas have the potential to influence the control of movement at both the cortical and the spinal levels.

The cortical regions included within our definition of the premotor cortex differ from those of others in several important respects. The original designation of the premotor cortex as a single cortical field (Fulton, 1935) included regions that we now consider separate premotor areas (SPcS, SMA, and CMAd). In addition, the APA, CMAr, and CMAv were excluded from the original definition by cytoarchitectonic criteria. Conversely, the original definition of the premotor cortex included a rostral portion of area 6 (i.e., the lateral part of area $6 \mathrm{aB}$ of Vogt and Vogt, 1919; 6DR of Barbas and Pandya, 1987). Because this cortical region lacks substantial direct projections to either the primary motor cortex or the spinal cord (e.g., Matsumura and Kubota, 1979; Muakkassa and Strick, 1979; Godschalk et al., 1984; Strick, 1985; Barbas and Pandya, 1987; Ghosh et al., 

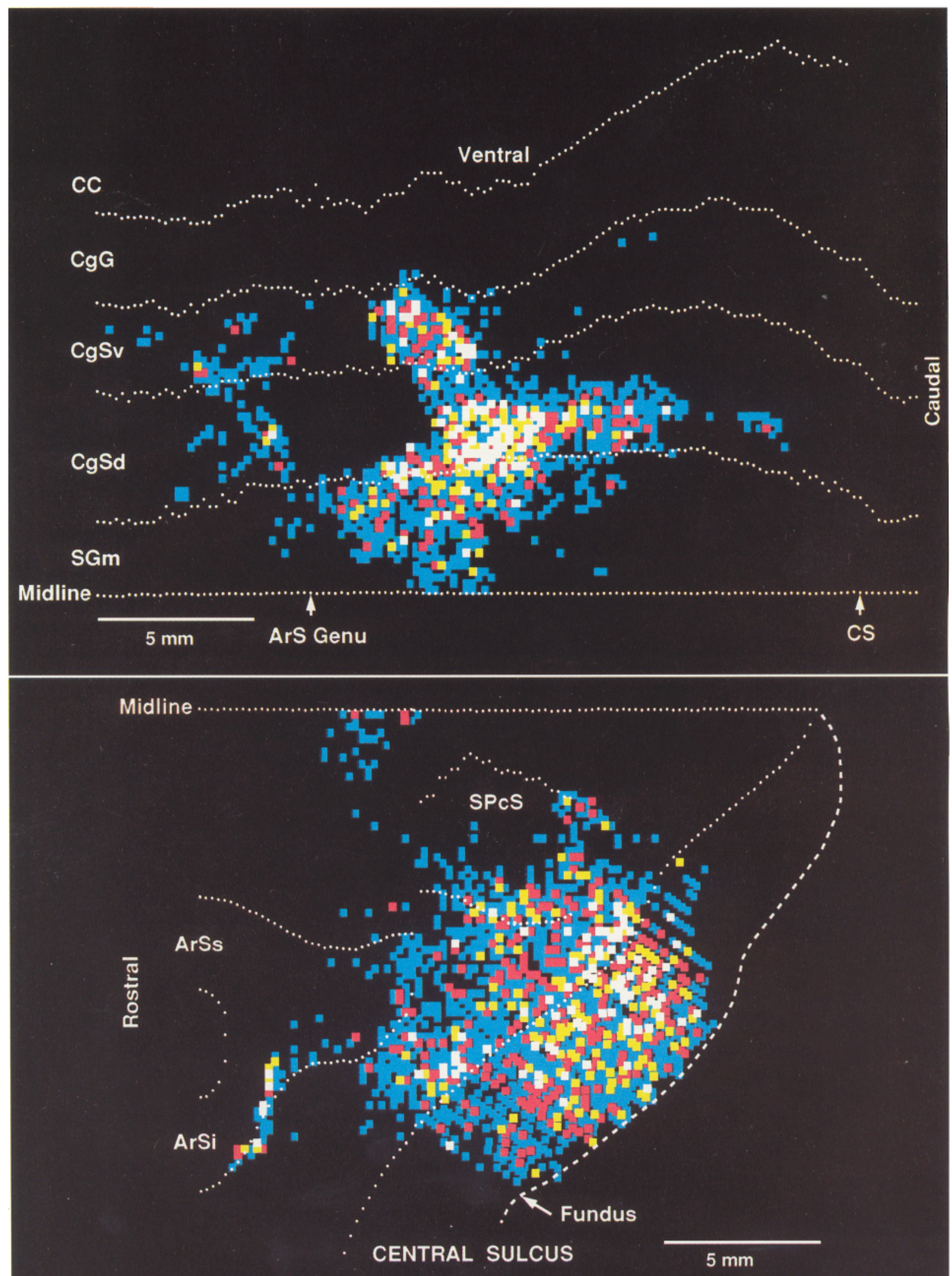

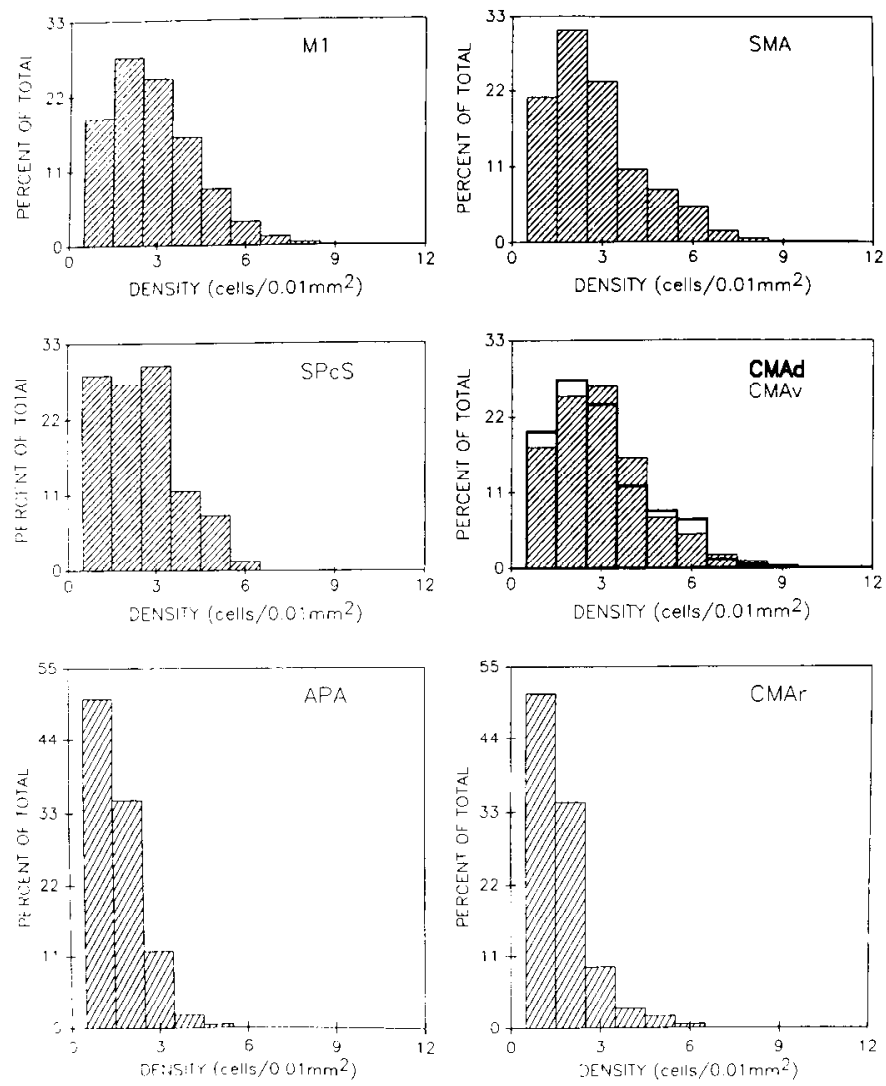

Figure 13. Histograms of density distribution of corticospinal neurons in motor areas of frontal lobe. The data in this figure are from the animal illustrated in Figures 8-12 (WGA-HRP into C4-T2). Cell counts were determined for $200-\mu \mathrm{m}$ bins along every fourth coronal section. $M I$, primary motor cortex.

1987; Dum and Strick, 1990), we believe that it should no longer be considered functionally as part of the premotor cortex.

It is important to note that our definition of the premotor cortex does not include all the cortical regions in the frontal lobe that may be associated with the control of limb movement. There are considerable data that suggest that the prefrontal cortex is involved in the planning, initiation, facilitation, and inhibition of motor responses (for review, see Goldman-Rakic, 1987; Fuster, 1989). In fact, some neurons in the prefrontal cortex display changes in activity that are tightly locked to limb movements (e.g., Kubota and Niki, 1971; Niki, 1974a,b; Watanabe, 1986). Furthermore, widespread areas of the frontal lobe

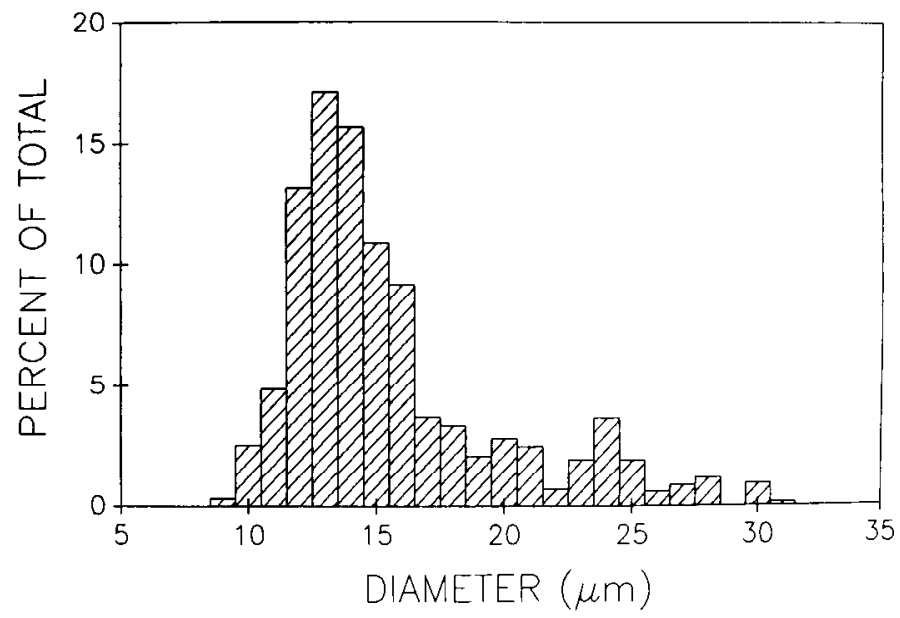

Figure 14. Soma diameters of corticospinal neurons in arm representations of motor areas in frontal lobe. The data in this figure are from the animal illustrated in Figures 8-12 (WGA-HRP into C4-T2). These measurements represent the average of the maximum and minimum soma diameter. Large neurons in this sample were defined as those measuring more than $19 \mu \mathrm{m}$.

contribute to basal ganglia and cerebellar loops (e.g., Brooks and Thach, 1981; Glickstein et al., 1985; Alexander et al., 1986; Goldman-Rakic, 1987) and, through these connections, may participate in the generation and control of movement. We suggest that the term "premotor cortex" should be reserved for those cortical areas that have the unique potential to influence directly motor output at the level of both the primary motor cortex and the spinal cord. Other cortical areas that do not fit this definition but clearly have a function in the generation and control of limb movement might be included within the broad term "nonprimary motor areas" (Wise et al., 1990).

\section{Location of the individual premotor areas}

An important consequence of the present study is a more complete definition of the location and extent of the "arm" representation in the premotor cortex. In total, the premotor cortex appears to contain at least 6 arm areas. In this section, we will briefly review the anatomical and physiological evidence that distinguishes these areas from each other and from the primary motor cortex.

One of the premotor areas is the APA, which is largely buried in the caudal bank of the arcuate sulcus. Woolsey et al. (1952) included part of this region within the head and neck represen-

Figure 11. Density of corticospinal neurons in frontal lobe: medial wall of hemisphere. The data in this figure are from the animal illustrated in Figures 8-12 (WGA-HRP into C4-T2). The conventions used in this figure are the same as those for Figure 12. The genu of the arcuate sulcus $(A r S G e n u)$ and the junction of the central sulcus $(C S)$ with the midline are indicated by arrows. The number of cells per bin is color coded as follows: white, 6-11; yellow, 5; red, 4; blue, 2-3. Note that each of the premotor areas on the medial wall contains at least 1 high-density bin. The percentage of the total bins at each level is $5 \%$ (highest density level), $5 \%, 8 \%, 42 \%$ (lowest density level illustrated), and $40 \%$ (density level not illustrated). Abbreviations are as in Figure 1.

Figure 12. Density of corticospinal neurons in frontal lobe: lateral surface. The data in this figure are from the animal illustrated in Figures 8-1 I (WGA-HRP into C4-T2). In this view, however, the SPcS was opened in a medial direction to display the density of labeled neurons within it The procedures used for dividing cortex into bins $(200 \mu \mathrm{m}$ on a side) and for correcting density counts for the angulation of the central sulcus are described in Materials and Methods. The number of cells per bin is color coded as follows: white, 6-10; yellow, 5; red, 4; blue, 2-3. Bins containing only 1 cell are not displayed. The white and the yellow levels contain the upper 10\% of the bins (high-density bins). Note that each premotor area contains at least 1 high-density bin. The density in the arcuate sulcus is not accurately displayed because this region was not flattened (see Figure 10). The percentage of the total bins at each level is $4 \%$ (highest density level), $6 \%, 12 \%, 45 \%$ (lowest density level illustrated), and $33 \%$ (density level not illustrated). See Materials and Methods and Results for further details. 

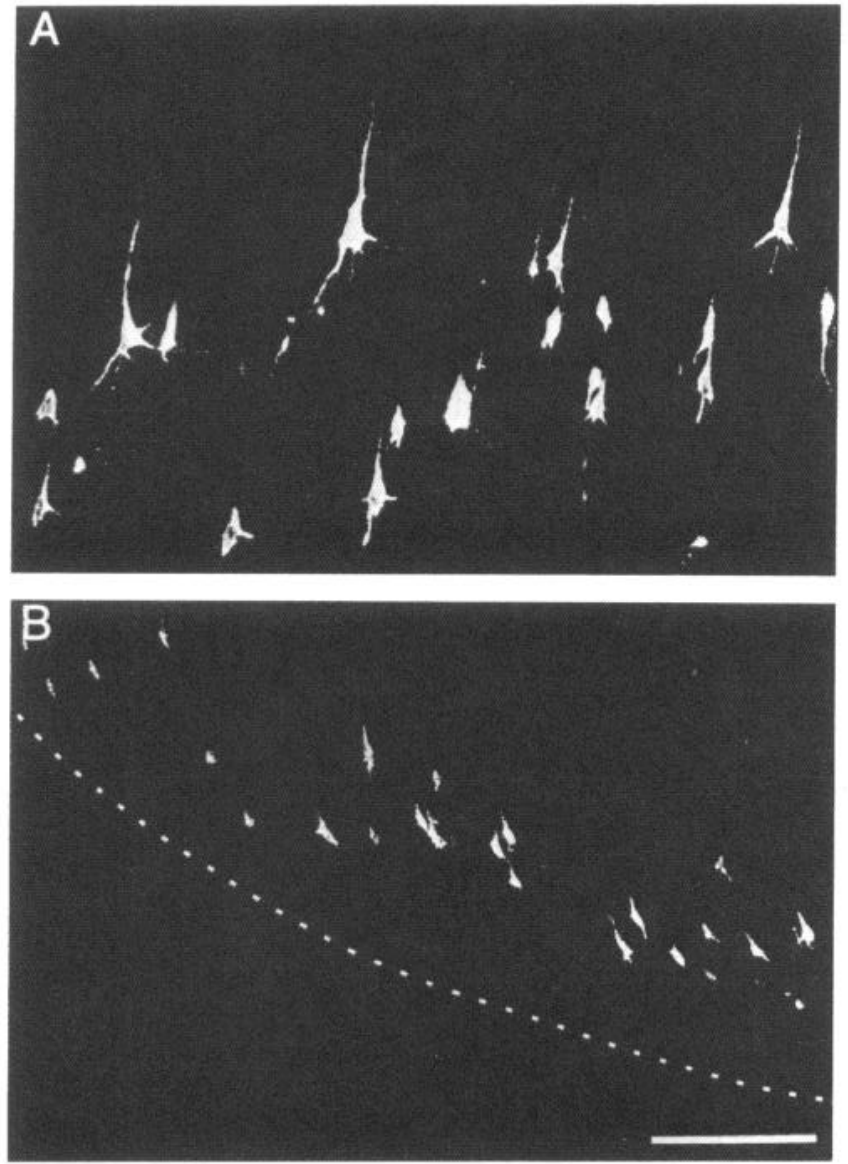

Figure 15. Corticospinal neurons. These photomicrographs were taken under dark-field/polarized light illumination. $A$, Corticospinal neurons located in the primary motor cortex. $B$, Corticospinal neurons located in the arm representation of the CMAv in area $23 \mathrm{c}$ on the ventral bank of the cingulate sulcus. The dashed line indicates the border between cortical layer VI and white matter. Scale bar, $250 \mu \mathrm{m}$.

tation of the primary motor cortex. However, movements of the digits and wrist have been evoked by intracortical stimulation in the caudal bank of the arcuate sulcus (Gentilucci et al., 1988). In addition, single neurons recorded in this sulcus in awake monkeys show changes in activity related to distal forelimb movements (Kubota and Hamada, 1978; Rizzolatti et al., 1981, 1988; Godschalk et al., 1985; Kurata and Tanji, 1986; Okano and Tanji, 1987). Anatomical studies provide further support for an arm representation in the APA by demonstrating that it projects to the arm area of the primary motor cortex and cervical segments of spinal cord (Matsumura and Kubota, 1979; Muakkassa and Strick, 1979; Godschalk et al., 1984; Martino and Strick, 1987; Dum and Strick, 1989). Anatomical studies also demonstrate that the corticocortical and thalamocortical connections of the APA, as well as its cytoarchitecture, are very different from those of the primary motor cortex (Schell and Strick, 1984; Matelli et al., 1985, 1986, 1989; Barbas and Pandya, 1987; Dum and Strick, 1990). These observations support our proposal that the APA is a distinct entity with a separate arm representation.

A second premotor area is located in the region of the cortex surrounding the SPcS. The existence of an arm representation and even a premotor area at this site has been controversial. In
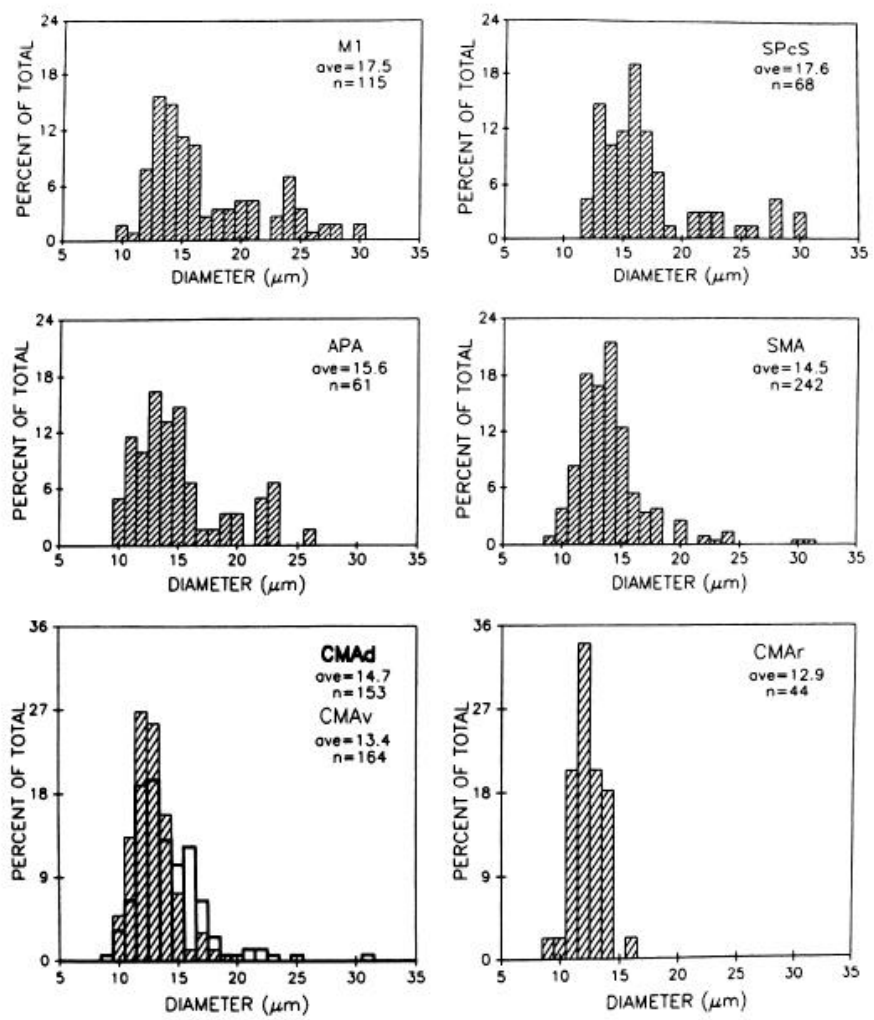

Figure 16. Soma diameters of corticospinal neurons in arm representations of premotor areas and primary motor cortex. The data in this figure are from the animal illustrated in Figures 8-12 (WGA-HRP into C4-T2). Note that large corticospinal neurons (soma diameter, $>19$ $\mu \mathrm{m}$ ) are found in 4 premotor areas (the SPcS, SMA, APA, and CMAd). $M 1$, primary motor cortex.

fact, some studies have included all or part of the SPcS within the primary motor cortex (e.g., Brodmann, 1905; von Bonin and Bailey, 1947; Woolsey et al., 1952; Sessle and Wiesendanger, 1982). Early physiological studies attributed the region around the SPcS with some unique characteristics and distinguished it from the primary motor cortex (e.g., Bucy and Fulton, 1933; Hines, 1937; Denny-Brown and Botterell, 1948). More recent anatomical and physiological data continue to indicate differences between this cortical area and the adjacent primary motor cortex. The thresholds for evoking movement in the SPcS are higher than those in the primary motor cortex (Kurata et al., 1985; Kurata, 1989). In awake trained monkeys, many neurons around the lateral edge of the SPcS change their activity in relation to distal forelimb movements and display "set-related" activity (Kurata et al., 1985; Kurata, 1989). In contrast, neurons with set-related activity are comparatively rare in the primary motor cortex. The SPcS and primary motor cortex also differ in that the SPcS has dense projections to the medullary reticular formation and the primary motor cortex does not (Keizer and Kuypers, 1989). The results of the present study indicate that large numbers of corticospinal neurons are found in and around the SPcS. This is the same region that contains a spatially separate group of labeled neurons following tracer injections into the arm area of the primary motor cortex (Muakkassa and Strick, 1979; Godschalk et al., 1984). Thus, there is both anatomical and physiological evidence to support the proposal that the cortex around the SPcS contains a premotor area with an arm representation distinct from the primary motor cortex. 


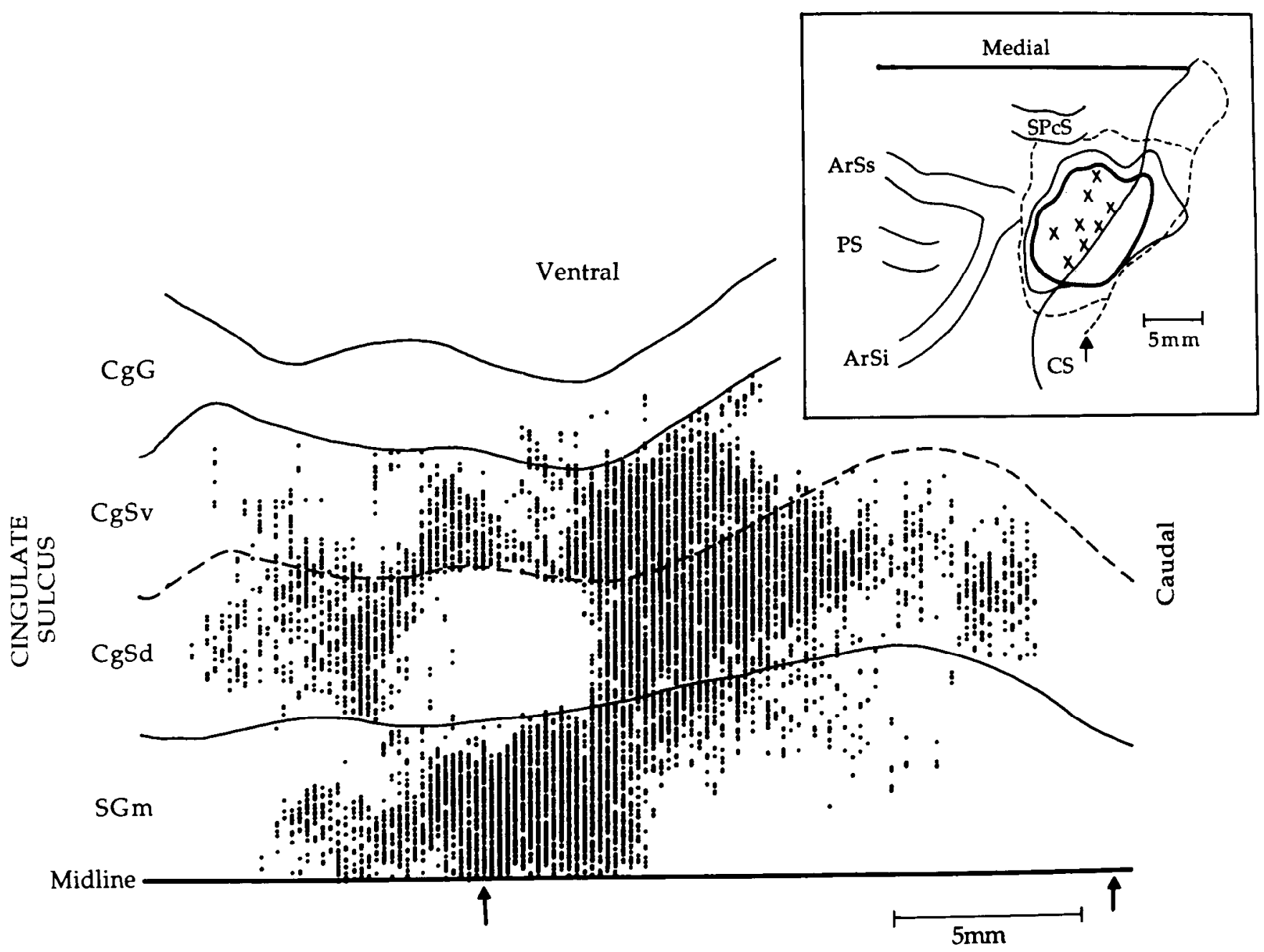

Figure 17. Map of cortical neurons projecting from premotor areas on medial wall to arm area of primary motor cortex. WGA-HRP was injected into the arm area of the primary motor cortex. The spread of tracer is indicated on the flattened reconstruction of the frontal lobe (inset at upper right $). X \mathrm{~s}$ mark the site of needle penetration into the cortex. A heavy line encircles the densest region of reaction product, and a lighter line indicates the "halo" that surrounded it. The dashed line encircles the region of almost continuous cell labeling that surrounded the injection site. Every fourth section was used to reconstruct the distribution of labeled neurons (dots) on the medial wall. The genu of the arcuate sulcus (left arrow) and the junction of the central sulcus with the midline (right arrow) are indicated. See Figures 1,6, and 8 for conventions and methods of reconstruction. Note that neurons projecting to the arm area of the primary motor cortex are located in the same regions that project to cervical segments of the spinal cord (cf. Fig. 8).

Four additional premotor areas, each of which has an arm representation, are located on the medial wall of the hemisphere (see also Muakkassa and Strick, 1979; Hutchins et al., 1988; Dum and Strick, 1989, 1990). One of these is the SMA, which has long been recognized as separate from the primary motor cortex (Penfield and Welch, 1951; Woolsey et al., 1952). There is a close correspondence between the location of corticospinal neurons in the SMA projecting to cervical segments of the spinal cord and other anatomical and physiological maps of arm representation on the superior frontal gyrus (e.g., Penfield and Welch, 1951; Brinkman and Porter, 1979; Matsumura and Kubota, 1979; Muakkassa and Strick, 1979; Macpherson et al., 1982; Godschalk et al., 1984; Mitz and Wise, 1987). For example, corticospinal neurons that project to cervical segments are most concentrated within regions of the superior frontal gyrus that lie 3-4 mm caudal to the genu of the arcuate sulcus. Apparently, this is the only site within the superior frontal gyrus where intracortical stimulation evokes arm movements (Macpherson et al., 1982; Mitz and Wise, 1987; Alexander and Crutcher, 1990). Thus, there is general agreement that a large part of the arm representation within the SMA lies on the superior frontal gyrus, caudal to the genu of the arcuate sulcus.

There is some disagreement, however, about the complete extent of arm representation within the SMA. Woolsey et al. (1952) indicated that part of its arm representation extended ventrally from the superior frontal gyrus into the depths of the dorsal bank of the cingulate sulcus. In other studies, stimulation in this region of the dorsal bank evoked movements of the hindlimb (Penfield and Welch, 1951; Macpherson et al., 1982; Mitz and Wise, 1987). In the present study, the portion of the dorsal bank of the cingulate sulcus that lies ventral to the SMA arm representation on the superior frontal gyrus lacked projections either to cervical segments of the spinal cord or to the arm area of the primary motor cortex. Indeed, we and others have found that this region of the dorsal bank projects to lumbar segments of the spinal cord (Biber et al., 1978; Murray and 

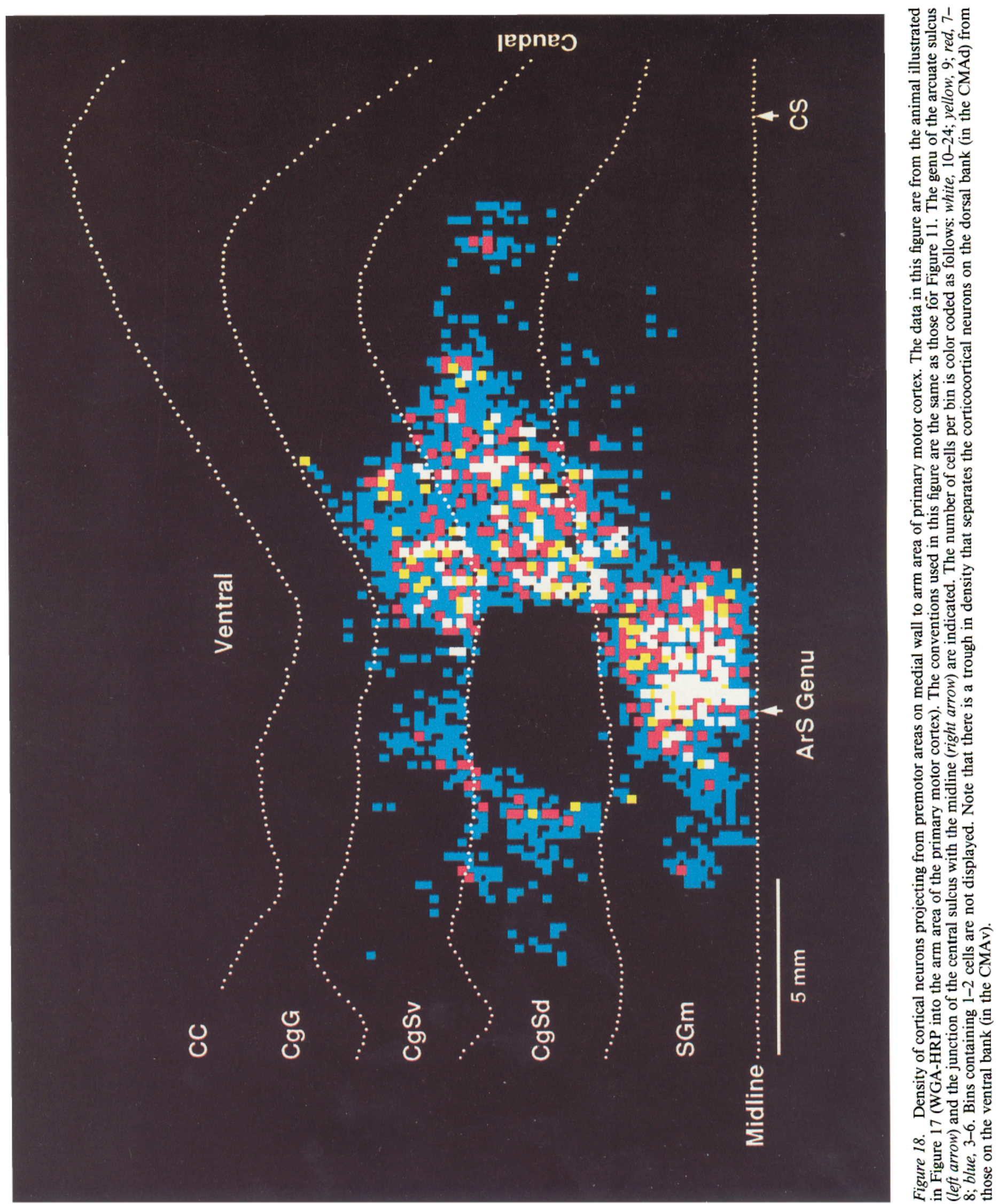
Coulter, 1981; Macpherson et al., 1982; He et al., 1989). Thus, most of the available evidence suggests that the arm area of the SMA is largely confined to the superior frontal gyrus.

Two additional premotor areas, the CMAd and the CMAv, are located more caudally in the cingulate sulcus (see also Hutchins et al., 1988; Dum and Strick, 1989, 1990). Although our initial description treated these areas as a single cortical region (Hutchins et al., 1988), they span 2 distinct cytoarchitectonic fields. The CMAv resides in area 23c, and the CMAd resides in arca $6 \mathrm{c}$ (sce also von Bonin and Bailey, 1947; Vogt et al., 1987). In addition, the present study demonstrates that tracer injections into either the arm area of the primary motor cortex or cervical segments of spinal cord result in a high-density region of labeled neurons in the CMAd that is separated from that in the CMAv by a trough in labeled neurons. Furthermore, recent anatomical studies in our laboratory indicate that the subcortical and cortical projections to the 2 portions of the CMAc differ. For example, the CMAv, but not the CMAd, is interconnected with regions of the prefrontal cortex adjacent to the principal sulcus (Lu and Strick, 1990). The CMAv receives thalamic input from a caudal portion of the ventralis lateralis pars caudalis (VLc; Holsapple and Strick, 1989), a site of termination of cerebellar efferents (e.g., Percheron, 1977; Stanton, 1980; Kalil, 1981; Asanuma et al., 1983a,b). In contrast, the CMAd receives thalamic input from a portion of the ventralis laterlis par oralis (VLo) (Holsapple and Strick, 1989), a site of termination of pallidal efferents (e.g., Nauta and Mehler, 1966; Kuo and Carpenter, 1973; Kim et al., 1976; DeVito and Anderson, 1982). Thus, the available anatomical evidence supports the division of the CMAc into 2 premotor areas with separate arm representations.

Before proceeding, we would like to clarify a potential source of confusion about the CMAd. Woolscy ct al. (1952) considered this region to be a part of the SMA and indicated that it contained the representation of the proximal hindlimb (cf. Fig. 8, 17 with Woolsey et al., 1952, p 255, their Fig. 132B). However, using stimulation techniques comparable to Woolsey et al. (1952), Penfield and Welch (1951) evoked forelimb movements when they stimulated this approximate location on the dorsal bank of the cingulate sulcus (see, e.g., their Fig. 5, points 19, 52). We have confirmed prior observations that the architecture in the region of the CMAd differs from that of the SMA on the adjacent superior frontal gyrus (Mauss, 1908; Brodmann, 1909; von Bonin and Bailey, 1947; Toyoshima and Sakai, 1982). The large injections of tracer employed in the present study produced a region of labeled neurons that continued uninterrupted from the SMA to the CMAd. However, after tracer injections limited to lower cervical segments of the spinal cord or small injections into the arm representation of the primary motor cortex, there was a clear separation between the labeled neurons in the SMA and those in the CMAd (Strick, 1985; Hutchins et al., 1988; Dum and Strick, 1989, 1990; He et al., 1989). Taken together, these observations support our conclusion that the CMAd contains an arm representation distinct from that in the SMA.

The fourth premotor area and arm representation on the medial wall is located in the banks of the cingulate sulcus at levels rostral to the genu of the arcuate sulcus. This premotor area resides in a subfield of area 24 and was termed "CMAr" (Hutchins et al., 1988; Dum and Strick, 1990). Besides its connections with the primary motor cortex and spinal cord (Künzle, 1978; Leichnetz, 1986; Martino and Strick, 1987; Dum and Strick, 1990), little is known about the CMAr. However, in awake primates, Shima et al. (1991) have recorded the activity of neurons in the CMAr, CMAd, and CMAv. All 3 regions contained neurons whose activities were related to the execution of stimulus-triggered and self-paced movements of the distal forelimb. Many neurons in the cingulate motor areas $(60 \%)$ discharged before distal muscles became active in the task. Compared to the CMAd and CMAv, the CMAr contained a higher concentration of neurons that were preferentially active during selfpaced movements. Also, neurons with changes in activity long before the onset of self-paced movements $(0.5-2.0 \mathrm{sec})$ were particularly abundant in the CMAr. These observations provide additional evidence for distinguishing the CMAr from the more caudal premotor areas in the cingulate sulcus. Furthermore, they support our proposal that the cingulate motor areas are involved in the generation of arm movements.

\section{Contribution of the premotor areas to the corticospinal sustem}

One of the major results of the present study is our finding that the total number of corticospinal neurons in the arm representations of the premotor areas equals or exceeds the total number in the arm representation of the primary motor cortex. Furthermore, the premotor areas collectively comprise more than $60 \%$ of the cortical area in the frontal lobe that projects to the spinal cord. As much as $40 \%$ of the corticospinal neurons in the frontal lobe originate from the premotor areas on the medial wall of the hemisphere (i.e., SMA, CMAd, CMAv, and CMAr). Up to $18 \%$ of the corticospinal system in the frontal lobe originates from a single premotor area, the SMA. These observations are consistent with the findings of Russell and DeMyer (1961) that, on average, $29 \%$ of the pyramidal tract at the medullary level originates from area 6 and $31 \%$ originates from area 4.

Individually, the contribution of each premotor area to the corticospinal system may not appear substantial. For example, the area of the arm representation in the SMA is approximately half the area of the arm representation in the primary motor cortex. Also, the number of corticospinal neurons in the SMA is less than half that of the primary motor cortex. However, several studies suggest that the primary motor cortex is actually composed of functional subdivisions. In the squirrel monkey, the arm area of the primary motor cortex contains 2 spatially separate representations of the hand, which receive different concentrations of "deep" and "cutaneous" afferent input (Strick and Preston, 1978a,b, 1982a,b). A similar organization may be present in the arm and leg representations of macaques (Lamour et al., 1980; Lemon, 1981; Wise and Tanji, 1981; Sato and Tanji, 1989). Thus, it may be more appropriate to compare individual premotor areas with one of the subdivisions of the primary motor cortex.

A second major result of the present study is that each of the premotor areas contains local regions that have a high density of corticospinal neurons. In fact, the overall density distributions of corticospinal neurons in the CMAd and CMAv were not significantly different from that in the primary motor cortex. These observations suggest that the local density of corticospinal output from some of the premotor areas is comparable to that from the primary motor cortex.

These 2 major results emphasize that a substantial component of the corticospinal system originates from the premotor areas in the frontal lobe. Each of the premotor areas has direct access to the spinal cord, and, as a consequence, each has the potential to influence the generation and control of movement independently of the primary motor cortex. This conclusion contrasts 
sharply with the classical viewpoint that the primary motor cortex is the main source of motor signals in the corticospinal tract. In fact, some have viewed the primary motor cortex as the "upper motoneuron" or the "final common pathway" for the cortical control of movement (for review, see Phillips and Porter, 1977; Kuypers, 1981; Hepp-Reymond, 1988). This view is clearly reflected in Fulton's statement that area 4 "serves as a funnel through which highly organized movement-patterns are ultimately discharged" (Fulton, 1949, p 393). It is now clear that the primary motor cortex is only one of a number of cortical areas that have the potential to influence motor function at the spinal level.

\section{Influence on spinal mechanisms}

Large corticospinal neurons are thought to have a special role in movement initiation, in part because they provide a substantial portion of the monosynaptic input from the motor cortex to motoneurons (for review, see Phillips and Porter, 1977; Evarts, 1981). We found that "large" corticospinal neurons (mean diameter, $>19 \mu \mathrm{m}$ ) are present not only in the primary motor cortex, but also in 4 out of 6 premotor areas. Although the primary motor cortex contains the highest percentage of large corticospinal neurons $(31 \%)$, these ncurons comprise as much as $20 \%$ of the population of corticospinal neurons originating from the APA and the SPcS. In this respect, the corticospinal system originating from the premotor areas differs from that in the parietal lobe, where few if any large corticospinal neurons are present (Jones and Wise, 1977; Murray and Coulter, 1981; Toyoshima and Sakai, 1982). The presence of large corticospinal neurons in the premotor areas is consistent with the hypothesis that these cortical regions participate in the actual generation of movement.

On the other hand, most (92\%) of the corticospinal neurons in the premotor areas are "small" (mean diameter, between 9 and $19 \mu \mathrm{m})$. Small neurons also make up the vast majority $(69 \%)$ of the corticospinal population in the primary motor cortex. I he small size of these neurons, however, should not preclude them from having an important function in motor control and/ or participating in the gencration of limb movement. The results of recent physiological studies in the primary motor cortex indicate that some presumably small corticospinal neurons with more slowly conducting axons make monosynaptic connections with motoneurons (Fetz and Cheney, 1980; Buys et al., 1986). Furthermore, some small corticospinal neurons change their activity before the onset of EMG activity and movement (e.g., Evarts, 1966; Fromm and Evarts, 1981).

The functional contribution of corticospinal projections from the premotor areas cannot be fully evaluated until the patterns of spinal termination of efferents from these areas are determined. Results from preliminary studies (R. P. Dum and P. L. Strick, unpublished observations; see also Brinkman, 1982; Cheema et al., 1983) suggest that the corticospinal projections from the CMAd, CMAv, and SMA terminate most heavily in the intermediate zone of the spinal cord. In this respect, the pattern of termination of corticospinal projections from the premotor areas is like that from the primary motor cortex (Kuypers, 1960; Liu and Chambers, 1964; Kuypers and Brinkman, 1970; Cheema et al., 1984; Ralston and Ralston, 1985). The intermediate zone of the spinal cord contains many interneurons that project to motoneurons (for review, see Kuypers, 1981). Thus, the limited evidence available suggests that the cortico- spinal projections from the premotor areas have direct access to spinal cord mechanisms concerned with motor output.

\section{Functional implications of spinal access for the premotor areas}

Our observations suggest a new perspective on the involvement of the premotor areas in the generation and control of movement. As noted in the introductory remarks, classically, the cortical motor areas were viewed as organized in a hierarchical manner in which the premotor cortex occupied a level superior to that of the primary motor cortex. The generation of motor commands was thought to proceed in a serial fashion from the premotor cortex to the primary motor cortex and hence to the spinal cord (e.g., Campbell, 1905; Fulton, 1949; for recent reviews, see Wiesendanger, 1981; Freund and Hummelsheim, 1985; Wise, 1985). Thus, the premotor areas have been thought to influence the control of movement (especially that of distal body parts) primarily through their connections with the primary motor cortex.

The results of studies on the origin of subcortical inputs to the premotor areas (reviewed in the introductory remarks) began to raise questions about a strictly serial model of motor control at the cortical level (e.g., Schell and Strick, 1984; Wiesendanger and Wiesendanger, 1985a,b; Matelli et al., 1989; Orioli and Strick, 1989). For example, Schell and Strick (1984) proposed that the arm area of the SMA is a target of pallidothalamic efferents, and in contrast, the arm area of the APA is a target of cerebellothalamic efferents. Furthermore, they suggested that the projections from the cerebellum and basal ganglia to the premotor areas were independent of those to the primary motor cortex. The organization of parietal lobe projections to the arm areas of the SMA, APA, and primary motor cortex appears to be organized along similar lines (Galyon and Strick, 1985; Matelli et al., 1986; Dum and Strick, 1990). Briefly, each cortical area receives its most substantial input from a different region of the parietal lobe. Some parietal lobe regions project to both the primary motor cortex and either the APA or the SMA. However, no parietal lobe region has substantial projections to both premotor areas. Thus, the premotor areas receive a unique pattern of input from the parietal lobe, as well as from subcortical motor centers

The parallel and independent nature of the inputs to the cortical motor areas led us to hypothesize that the premotor areas function, in part, at the same hierarchical level as the primary motor cortex (Strick, 1988; Dum and Strick, 1990). The results of the present study add further support for this hypothesis by demonstrating that, like the primary motor cortex, each premotor area has direct access to the spinal cord. Indeed, our findings indicate that the corticospinal system from the premotor areas is numerically as substantial as that from the primary motor cortex. Thus, we propose that the generation and control of movement at the cortical level are mediated by parallel outputs from the premotor areas, as well as from the primary motor cortex.

By making this proposal, we do not mean to imply that the premotor areas influence the control of movement only via their corticospinal projections; nor do we mean to imply that the premotor areas function only in parallel with the primary motor cortex. The direct connections of the premotor areas with the primary motor cortex and their participation in cerebellar and basal ganglia loops have long been thought to provide the anatomical substrate for serial interactions between the premotor 
areas and the primary motor cortex (e.g., Brooks and Thach, 1981; Ito, 1984; Glickstein et al., 1985; Alexander et al., 1986). Instead, we wish to emphasize a viewpoint that has been somewhat neglected, namely, that the premotor areas have parallel outputs directly to the spinal cord that may be independent of these serial interactions.

Many different functions have been attributed to the premotor cortex (e.g., visual guidance of movement, bimanual coordination, movement sequencing, preparation for movement, building associations between motor responses and arbitrary stimuli, postural mechanisms to support movement and trajectory control; e.g., Moll and Kuypers, 1977; Roland et al., 1980; Brinkman, 1984; Freund and Hummelsheim, 1985; Passingham, 1988; Halsbad and Freund, 1990; for review, see Wiesendanger, 1981; Wise, 1985). Perhaps these diverse viewpoints on the function of the premotor cortex are appropriate and reflect the functions of all 6 of the premotor areas. Thus, it is possible that each of these premotor areas may be differentially involved in one or another of these aspects of motor behavior. In fact, one potential consequence of the parallel input-output organization we propose for the motor areas in the frontal lobe is that this anatomical arrangement may facilitate the concurrent processing of different aspects of movement. Now that the locations of the premotor areas have been more precisely identified in primates, it should be possible to use combined behavioral and physiological methods to examine the contributions of individual premotor areas to motor control.

\section{References}

Alexander GE, Crutcher MD (1990) Preparation for movement: neural representations of intended direction in three motor areas of the monkey. J Neurophysiol 64:133-150.

Alexander GE, DeLong MR, Strick PL (1986) Parallel organization of functionally segregated circuits linking basal ganglia and cortex. Annu Rev Neurosci 9:357-381.

Asanuma C, Thach WT, Jones EG (1983a) Distribution of cerebellar terminations and their relation to other afferent terminations in the ventral lateral thalamic region of the monkey. Brain Res 286:237265.

Asanuma C. Thach WT, Jones EG (1983b) Anatomical evidence for segregated focal groupings of efferent cells and their terminal ramifications in the cerebellothalamic pathway of the monkey. Brain Res 286:267-297.

Barbas H, Pandya DN (1987) Architecture and frontal cortical connections of the premotor cortex (area 6) in the rhesus monkey. J Comp Neurol 256:211-228.

Bibcr MP, Kncisley LW, LaVail JH (1978) Cortical neurons projecting to the cervical and lumbar enlargements of the spinal cord in young and adult rhesus monkeys. Exp Neurol 59:492-508.

Brinkman C (1982) Supplementary motor area (SMA) and premotor area (PM) of the monkey's brain: distribution of degeneration in the spinal cord after unilateral lesions. Neurosci Lett 8:36.

Brinkman C (1984) Supplementary motor area of the monkey's cerebral cortex: short- and long-term deficits after unilateral ablation and the effects of subsequent callosal section. J Neurosci 4:918-929.

Brinkman C, Porter R (1979) Supplementary motor area in the monkey: activity of neurons during performance of a learned motor task. J Neurophysiol 42:681-709.

Brodmann K (1905) Beitraege zur histologischen Lokalisation der Grosshirnrinde. III. Mitteilung: Die Rindenfelder der niederen Affen. J Psychol Ncurol (Leipzig) 4:177-226.

Brodmann K (1909) Vergleichende Lokalisationslehre der Grosshirnrinde in ihren Prinzipien dargestellt auf Grund des Zellenbaues. Leipzig: Barth.

Brooks VB, Thach WT (1981) Cerebellar control of posture and movement. In: Handbook of physiology, Sec I, The nervous system, Vol II, Motor control, Pt I (Brooks VB, ed), pp 877-946. Bethesda, MD: American Physiological Society.
Brushart TM, Mesulam MM (1980) Transganglionic demonstration of central sensory projections from skin and muscle with HRP-lectin conjugates. Neurosci Lett 17:1-6.

Bucy PC, Fulton JF (1933) Ipsilateral representation in the motor and premotor cortex of monkeys. Brain 56:318-342.

Buys EJ, Lemon RN, Mantel GW, Muir RB (1986) Selective facilitation of different hand muscles by single corticospinal neurones in the conscious monkey. J Physiol (Lond) 381:529-549.

Campbell AW (1905) Histological studies on the localization of cerebral function. Cambridge: Cambridge UP.

Catsman-Berrevoets CE, Kuypers HGJM (1976) Cells of origin of cortical projections to dorsal column nuclei, spinal cord and bulbar medial reticular formation in the rhesus monkey. Neurosci Lett 3 : 245-252.

Cheema S, Whitsel BL, Rustioni A (1983) Corticospinal projections from pericentral and supplementary cortices in macaques as revealed by anterograde transport of horseradish peroxidase. Neurosci Lett 14: 62.

Cheema SS, Rustioni A, Whitsel BL (1984) Light and electron microscopic evidence for a direct corticospinal projection to superficial laminae of the dorsal horn in cats and monkeys. J Comp Neurol 225: 276-290.

Denny-Brown D, Botterell EH (1948) The motor functions of the agranular frontal cortex. Assoc Res Nerv Ment Dis 276:235-345.

DeVito JL, Anderson ME (1982) An autoradiographic study of efferent connections of the globus pallidus in Macaca mulatta. Exp Brain Res 46:107-117.

Dum RP, Strick PL (1988) Origin and density of corticospinal (CST) projections from the premotor areas of macaques. Soc Neurosci Abstr 14:821.

Dum RP, Strick PL (1989) Corticospinal projections from the motor areas in the frontal lobe. In: Neural programming (Ito M, ed), pp 4963. Tokyo: Japan Scientific Societies Press.

Dum RP, Strick PL (1990) Premotor areas: nodal points for parallel efferent systems involved in the central control of movement. In: Motor control: concepts and issues (Humphrey DR Freund H-J, eds), pp 383-397. London: Wiley.

Evarts EV (1966) Pyramidal tract activity associated with a conditioned hand movement in the monkey. J Neurophysiol 29:10111027.

Evarts EV (1981) Role of motor cortex in voluntary movements in primates. In: Handbook of physiology, Sec I, The nervous system, Vol II, Motor control, Pt I (Brooks VB, ed), pp 1083-1120. Bethesda, MD: American Physiological Society.

Fetz EE, Cheney PD (1980) Postspike facilitation of forelimb muscle activity by primate corticomotoneuronal cells. J Neurophysiol 44: 751-772.

Foerster $O$ (1936) The motor cortex in man in light of Hughlings Jackson's observations. Brain 59:135-159.

Freund H-J, Hummelsheim H (1985) Lesions of premotor cortex in man. Brain 108:697-733

Fromm C, Evarts EV (1981) Relation of size and activity of motor cortex pyramidal tract neurons during skilled movements in the monkey. J Neurosci 1:453-460.

Fulton JF (1935) Definition of the 'motor' and 'premotor' areas. Brain $58: 311-316$.

Fulton JF (1949) Physiology of the nervous system. New York: Oxford UP.

Fulton JF, Kennard MA (1934) A study of flaccid and spastic paralysis produced by lesions of the cerebral cortex in primates. Assoc Res Nerv Ment Dis 13:158-210.

Fuster JM (1989) The prefrontal cortex. New York: Raven.

Galyon DD, Strick PL (1985) Multiple and differential projections from the parietal lobe to the premotor areas of the primate. Soc Neurosci Abstr 11:1274.

Gentilucci M, Fogassi L, Luppino G, Matelli M, Camarda R, Rizzolatti $G$ (1988) Functional organization of inferior area 6 in the macaque monkey. I. Somatotopy and the control of proximal movements. Exp Brain Res 71:475-490.

Ghosh S, Brinkman C, Porter R (1987) A quantitative study of the distribution of neurons projecting to the precentral motor cortex in the monkey (M. fascicularis). J Comp Neurol 259:424-444.

Gibson AR, Hansma DI, Houk JC, Robinson FR (1984) A sensitive low artifact TMB procedure for the demonstration of WGA-HRP in the CNS. Brain Res 298:235-241. 
Glickstein M, May JG III, Mercier BE (1985) Corticopontine projection in the macaque: the distribution of labelled cortical cells after large injections of horseradish peroxidase in the pontine nuclei. $J$ Comp Neurol 235:343-359.

Godschalk M, Lemon RN, Kuypers HG, Ronday HK (1984) Cortical afferents and efferents of monkey postarcuate area: an anatomical and electrophysiological study. Exp Brain Res 56:410-424.

Godschalk M, Lemon RN, Kuypers HG, van der Steen J (1985) The involvement of monkey premotor cortex neurones in preparation of visually cued arm movements. Behav Brain Res 18:143-157.

Goldman-Rakic PS (1987) Motor control function of the prefrontal cortex. In: Motor areas of the cerebral cortex (Ciba Foundation symposium 132), pp 187-200. Chichester: Wiley.

Gonatas NK, Harper C, Mizutani T, Gonatas JO (1979) Superior sensitivity of conjugates of horseradish peroxidase with wheat germ agglutinin for studies of retrograde axonal transport. J Histochem Cytochem 27:728-734.

Halsband U, Freund H-J (1990) Premotor cortex and conditional motor learning in man. Brain 113:207-222.

Hartman CG, Straus WL (1933) The anatomy of the rhesus monkey. New York: Hafner.

He SQ, Dum RP, Strick PL (1989) Premotor areas on the medial wall of the hemisphere: corticospinal projections to cervical and lumbosacral cord. Soc Neurosci Abstr 15:282.

Hepp-Reymond MC (1988) Functional organization of motor cortex and its participation in voluntary movements. In: Comparative primate biology, Vol 4, Neurosciences, pp 501-624. New York: Liss.

Hill WCO (1974) Primates: comparative anatomy and taxonomy, Vol 7. New York: Wiley.

Hines M (1937) The "motor" cortex. Bull Johns Hopkins Hosp 60: 313-336.

Holsapple JW, Strick PL (1989) Premotor areas on the medial wall of the hemisphere: input from ventrolateral thalamus. Soc Neurosei Abstr 15:282.

Huarig CS, Sirisko MA, Hiraba H, Murray GM, Sessle BJ (1988) Organization of the primate face motor cortex as revealed by intracortical microstimulation and electrophysiological identification of afferent inputs and corticobulbar projections. J Neurophysiol 59:796-818.

Humphrey DR (1979) On the cortical control of visually directed reaching: contribution by nonprecentral motor areas. In: Posture and movement (Talbot RE, Humphrey DR, eds), pp 51-112. New York: Raven.

Hutchins KD, Martino AM, Strick PL (1988) Corticospinal projections from the medical wall of the hemisphere. Exp Brain Res 71: $667-672$.

Ito M (1984) The cerebellum and neural control. New York: Raven.

Jacobsen CF (1934) Influence of motor and premotor lesions upon retention of acquired skilled movements in monkey and chimpanzees. Assuc Res Nerv Ment Dis 13:225-247.

Jacobsen CF (1935) Functions of the frontal association areas in primates. Arch Neurol Psychiatry 33:558-569.

Johnson PB, Angelucci A, Ziparo RM, Minciacchi D, Bentivoglio M, Caminiti R (1989) Segregation and overlap of callosal and association neurons in frontal and parietal cortices of primates: a spectral and coherency analysis. J Neurosci 9:2313-2326.

Jones EG, Wise SP (1977) Size, laminar and columnar distribution of efferent cells in the sensory-motor cortex of monkeys. I Comp Neurol 175:391-438.

Kalil K (1981) Projections of the cerebellar and dorsal column nuclei upon the thalamus of the rhesus monkey. J Comp Neurol 195:2550.

Keizer K, Kuypers HG (1989) Distribution of corticospinal neurons with collaterals to the lower brain stem reticular formation in monkey (Macaca fascicularis). Exp Brain Res 74:311-318.

Kim R, Nakano K, Jayaraman A, Carpenter MB (1976) Projections of the globus pallidus and adjacent structures: an autoradiographic study in the monkey. J Comp Neurol 169:263-289.

Kubota K. Hamada I (1978) Visual tracking and neuron activity in the post-arcuate area in monkeys. J Physiol (Paris) 74:297-312.

Kubota K, Niki H (1971) Prefrontal cortical unit activity and delayed alternation performance in monkeys. J Neurophysiol 34:337-347.

Künzle H (1978) Cortico-cortical efferents of primary motor and somatosensory regions of the cerebral cortex. Neuroscience 3:25-39.

Kuo JS, Carpenter MB (1973) Organization of pallidothalamic projections in rhesus monkey. J Comp Neurol 151:201-236.
Kurata K (1989) Distribution of neurons with set- and movementrelated activity before hand and foot movements in the premotor cortex of rhesus monkeys. Exp Brain Res 77:245-256.

Kurata K, Tanji J (1986) Premotor cortex neurons in macaques: activity before distal and proximal forelimb movements. J Neurosci 6: 403-411.

Kurata K, Okano K, Tanji J (1985) Distribution of ncurons related to a hindlimb as opposed to forelimb movement in the monkey premotor cortex. Exp Brain Res 60:188-191.

Kuypers HGJM (1960) Central cortical projections to motor and somato-sensory cell groups. Brain 83:161-184.

Kuypers HGJM (1981) Anatomy of the descending pathways. In: Handbook of physiology, Sec I, The nervous system, Vol II, Motor control, Pt I (Brooks VB, ed), pp 597-666. Bethesda, MD: American Physiological Society.

Kuypers HGJM, Brinkman J (1970) Precentral projections to different parts of the spinal intermediate zone in the rhesus monkey. Brain Res 24:29-48.

Kwan HC, MacKay WA, Murphy JT, Wong YC (1978) Spatial organization of precentral cortex in awake primates. II. Motor outputs. J Ncurophysiol 41:1120-1131.

Lamour $Y$, Jennings VA, Solis H (1980) Functional characteristics and segregation of cutaneous and non-cutaneous neurons in monkey precentral motor cortex (MI). Soc Neurosci Abstr 6:158.

Leichnetz GR (1986) Afferent and efferent connections of the dorsolateral precentral gyrus (area 4, hand/arm region) in the macaque monkey, with comparisons to area 8. J Comp Neurol 254:460-492.

Lemon RN (1981) Functional properties of monkey motor cortex neurones receiving afferent input from the hand and fingers. J Physiol (Lond) 311:497-519.

Liu CN, Chambers WW (1964) An experimental study of the corticospinal system in the monkey (Macaca mulatta). J Comp Neurol 123: 257-284

Lu MT, Strick PL (1990) Prefrontal connections with the premotor areas. Soc Neurosci Abstr 16:241.

Macpherson JM, Marangoz C, Miles TS, Wiesendanger M (1982) Microstimulation of the supplementary motor area (SMA) in the awake monkey. Exp Brain Res 45:410-416.

Martino AM, Strick PL (1987) Corticospinal projections originate from the arcuate premotor area. Brain Res 404:307-312.

Matelli M, Luppino G, Rizzolatti G (1985) Patterns of cytochrome oxidase activity in the frontal agranular cortex of the macaque monkey. Behav Brain Res 18:125-136.

Matelli M, Camarda R, Glickstein M, Rizzolatti G (1986) Afferent and efferent projections of the inferior area 6 in the macaque monkey. J Comp Neurol 251:281-298.

Matelli M, Luppino G, Fogassi L, Rizzolatti G (1989) Thalamic input to inferior area 6 and area 4 in the macaque monkey. J Comp Neurol 280:468-488.

Matsumura M, Kubota K (1979) Cortical projection to hand-arm motor area from post-arcuate area in macaque monkeys: a histological study of retrograde transport of horseradish peroxidase. Neurosci Lett 11:241-246.

Mauss T (1908) Die faserarchitektonische Gliederung der Grosshirnrinde bei niederen Affen. J Psychol Neurol (Leipzig) 13:263-325.

McGuinness E, Sivertsen D, Allman JM (1980) Organization of the face representation in macaque motor cortex. J Comp Neurol 193: 591-608.

Mesulam MM (1982) Tracing neural connections. New York: Wiley.

Mitz AR, Wise SP (1987) The somatotopic organization of the supplementary motor area: intracortical microstimulation mapping. $\mathrm{J}$ Neurosci 7:1010-1021.

Moll L, Kuypers IIG (1977) Premotor cortical ablations in monkeys: contralateral changes in visually guided reaching behavior. Science 198:317-319.

Muakkassa KF, Strick PL (1979) Frontal lobe inputs to primate motor cortex: evidence for four somatotopically organized 'premotor' areas. Brain Res 177:176-182.

Murray EA, Coulter JD (1981) Organization of corticospinal neurons in the monkey. J Comp Neurol 195:339-365.

Nauta WJH, Mehler WR (1966) Projections of the lentiform nucleus in the monkey. Brain Res 1:3-42.

Niki H (1974a) Prefrontal unit activity during delayed alternation in the monkey. I. Relation to direction of response. Brain Res 68:185196. 
Niki H (1974b) Prefrontal unit activity during delayed alternation in the monkey. II. Relation to absolute versus relative direction of response. Brain Res 68:197-204.

Nudo RJ, Masterton RB (1990) Descending pathways to the spinal cord. III. Sites of origin of the corticospinal tract. J Comp Neurol 296:559-583.

Okano K, Tanji J (1987) Neuronal activities in the primate motor fields of the agranular frontal cortex preceding visually triggered and self-paced movement. Exp Brain Res 66:155-166.

Orioli P, Strick PL (1989) Cerebellar connections with the motor cortex and the arcuate premotor area: an analysis employing retrograde transneuronal transport of WGA-HRP. J Comp Neurol 288: 612-626.

Pandya DN, Kuypers HG (1969) Corticocortical connections in the rhesus monkey. Brain Res 13:13-36.

Pandya DN, Vignolo LA (1971) Intra- and interhemispheric projections of the precentral, premotor and arcuate areas in the rhesus Inonkey. Brain Res 26:217-233.

Passingham RE (1988) Premotor cortex and preparation for movement. Exp Brain Res 70:590-596.

Penfield W, Welch K (1951) Supplementary motor area of the cerebral cortex. Arch Neurol Psychiatry 66:289-317.

Percheron G (1977) The thalamic territory of cerebellar afferents and the lateral region of the thalamus of the macaque in stereotaxic ventricular coordinates. J Hirnforsch 18:375-400.

Phillips CG, Porter R (1977) Corticospinal neurons. Their role in movement (Monograph of the Physiological Society, No 34). London: Academic Press.

Ralston DD, Ralston HJ III (1985) The terminations of corticospinal tract axons in the macaque monkey. J Comp Neurol 242:325-337.

Rizzolatti G, Scandolara C, Matelli M, Gentilucci M (1981) Afferent properties of periarcuate neurons in macaque monkeys. I. Somatosensory responses. Behav Brain Res 2:125-146.

Rizzolatti G, Camarda R, Fogassi L, Gentilucci M, Luppino G, Matelli $M$ (1988) Functional organization of inferior area 6 in the macaque monkey. II. Area F5 and the control of distal movements. Exp Brain Res 71:491-507.

Roland PE, Larsen B, Lassen NA, Skinhoj E (1980) Supplementary motor area and other cortical areas in organization of voluntary movements in man. J Neurophysiol 43:118-136.

Rosene DL, Mesulam MM (1978) Fixation variables in horseradish peroxidase neurohistochemistry. I. The effects of fixation time and perfusion procedures upon enzyme activity. J Histochem Cytochem 26:28-39.

Rosene DL, Roy NJ, Davis BJ (1986) A cryoprotection method that facilitates cutting frozen sections of whole monkey brains for histological and histochemical processing without freezing artifact. J Histochem Cytochem 34:1301-1315.

Russell JR, DeMeyer W (1961) The quantitative cortical origin of pyramidal axons of Macaca mulatta. Neurology (Minneapolis) 11: 96-108.

Sato KC, Tanji J (1989) Digit-muscle responses evoked from multiple intracortical foci in monkey precentral motor cortex. J Neurophysiol 62:959-970

Schell GR, Strick PL (1984) The origin of thalamic inputs to the arcuate premotor and supplementary motor areas. J Neurosci 4:539560.

Sessle BJ, Wiesendanger M (1982) Structural and functional definition of the motor cortex in the monkey (Macaca fascicularis). J Physiol (Lond) 323:245-265.

Shima K, Aya K, Mushiake H, Inase M, Aizawa H, Tanji J (1991) Two movement-related foci in the primate cingulate cortex observed in signal triggered and self-paced forelimb movements. J Neurophysiol, in press.

Stanton GB (1980) Topographical organization of ascending cerebellar projections from the dentate and interposed nuclei in Macaca mulatta: an anterograde degeneration study. J Comp Neurol 190:699731.

Strick PL (1985) How do the basal ganglia and ccrcbcllum gain access to the cortical motor areas? Behav Brain Res 18:107-123.

Strick PL (1988) Anatomical organization of multiple motor areas in the frontal lobe: implications for recovery of function. In: Advances in neurology, Vol 47, Functional recovery in neurological disease (Waxman SG, ed) pp 293-312. New York: Raven.

Strick PL, Preston JB (1978a) Multiple representation in the primate motor cortex. Brain Res 154:366-370.

Strick PL, Preston JB (1978b) Sorting of somatosensory afferent information in primate motor cortex. Brain Res 156:364-368.

Strick PL, Preston JB (1982a) Two representations of the hand in area 4 of a primate. I. Motor output organization. J Neurophysiol 48:139 149.

Strick PL, Preston JB (1982b) Two representations of the hand in area 4 of a primatc. II. Somatosensory input organization. J Ncurophysiol 48:150-159.

Toyoshima K, Sakai H (1982) Exact cortical extent of the origin of the corticospinal tract (CST) and the quantitative contribution to the CST in different cytoarchitectonic areas. A study with horseradish peroxidase in the monkey. J Hirnforsch 23:257-269.

Vogt BA, Pandya DN, Rosene DL (1987) Cingulate cortex of the rhesus monkey. I. Cytoarchitecture and thalamic afferents. J Comp Neurol 262:256-270.

Vogt C, Vogt O (1919) Allgemeinere Ergebnisse unserer Hirnforschung. J Psychol Neurol (Leipzig) 25:277-462.

von Bonin G, Bailey P (1947) The neocortex of Macaca mulatta. Urbana: University of Illinois Press.

Watanabe M (1986) Prefrontal unit activity during delayed conditional Go/No-Go discrimination in the monkcy. II. Rclation to Go and NoGo responses. Brain Res 382:15-27.

Weinrich M, Wise SP (1982) The premotor cortex of the monkey. J Neurosci 2:1329-1345.

Wiesendanger M (1981) Organization of the secondary motor areas of the cerebral cortex. In: Handbook of physiology, Sec I, The nervous system, Vol II, Motor control, Pt II (Brooks VB, ed), pp 1121-1147. Bethesda, MD: American Physiological Society.

Wiesendanger M, Seguin JJ, Künzle H (1973) The supplementary motor area $-\mathbf{a}$ control system for posture. In: Control of posture and locomotion (Stein RB, Pearson KB, Smith RS, Redford JB, eds), pp 331-346. New York: Plenum.

Wiesendanger R, Wiesendanger M (1985a) The thalamic connections with medial area 6 (supplementary motor cortex) in the monkey (Macaca fascicularis). Exp Brain Res 59:91-104.

Wiesendanger R, Wiesendanger M (1985b) Cerebello-cortical linkage in the monkey as revealed by transcellular labeling with the lectin wheat germ agglutinin conjugated to the marker horseradish peroxidase. Exp Brain Res 59:105-117.

Wise SP (1985) The primate premotor cortex: past, present, and preparatory. Annu Rev Neurosci 8:1-19.

Wise SP, Tanji J (1981) Supplementary and precentral motor cortex: contrast in responsiveness to peripheral input in the hindlimb area of the unanesthetized monkey. J Comp Neurol 195:433-451.

Wise SP, Alexander GE, Altman JS, Brooks VB, Freund H-J, Fromm CJ, Humphrey DR, Sasaki K, Strick PL, Tanji J, Vogel S, Wiesendanger M (1990) What are the specific functions of the different cortical motor areas? In: Motor control: concepts and issues (Humphrcy DR, Freund II-J, eds). London: Wiley.

Woolsey CN, Settlage PH, Meyer DR, Sencer W, Hamuy TP, Travis AM (1952) Patterns of localization in precentral and "supplementary" motor area and their relation to the concept of a premotor area. Assoc Res Nerv Ment Dis 30:238-264. 